\title{
Lipschitz Continuity of Polyhedral Skorokhod Maps
}

\author{
P. Krejčí and A. Vladimirov
}

\begin{abstract}
We show that a special stability condition of the associated system of oblique projections (the so-called $\ell$-paracontractivity) guarantees that the corresponding polyhedral Skorokhod problem in a Hilbert space $X$ is solvable in the space of absolutely continuous functions with values in $X$. If moreover the oblique projections are transversal, the solution exists and is unique for each continuous input and the Skorokhod map is Lipschitz continuous in both spaces $C([0, T] ; X)$ and $W^{1,1}(0, T ; X)$. Also, an explicit upper bound for the Lipschitz constant is derived.
\end{abstract}

Keywords: Polyhedral Skorokhod problem, oblique reflections, Lipschitz continuity

AMS subject classification: 34A60, 47A30, 52B70, 90C25

\section{Introduction}

A class of models called Skorokhod problems is widely used in areas such as elastoplasticity, queueing theory, iterative optimization methods, mathematical economics (see references in $[2,4])$. Here we consider a particular case of polyhedral Skorokhod problems which can be described as follows.

A characteristic polyhedral set $Z$ is given in a Hilbert space $X$. For a given input function $u(t)$ defined in a time interval $[0, T]$ with values in $X$ we look for an output $x(t)$ with values in $Z$ such that the derivative $\dot{u}(t)-\dot{x}(t)$ (in an appropriate sense) belongs to a given reflection cone $\mathcal{R}(x(t))$ at the point $x(t)$. If the reflection rules, for each input $u$ in a suitable function space and for each initial condition $x_{0} \in Z$, determines a unique output $x$, then the mapping $\mathcal{S}:\left[x_{0}, u\right] \mapsto \xi:=u-x$ is called the Skorokhod map. Its analytical properties for different classes of inputs and in different metrics on the space of inputs and outputs play a crucial role in applications. In particular, the Lipschitz continuity of $\mathcal{S}$ in the metric of uniform convergence has been studied during the last

P. Krejčí: Acad. Sci. Czech Rep., Math. Inst., Žitná 25, CZ-11567 Praha 1, Czech Republic; krejci@math.cas.cz. Supported by the Deutsche Forschungsgemeinschaft (DFG) during the author's stay at WIAS Berlin.

A. A. Vladimirov: Inst. for Information Transmission, Bol'shoi Karetnyi 19, Moscow, Russia; vladim@iitp.ru, aav@redline.ru. This work has been done during the author's stay at WIAS Berlin and at TU München in December 1999 - March 2000, supported in part by DFG grant 436 RUS 17/116/99. Partially supported also by the Russian Federation for Fundamental Research, Grants 0001-0571 and 00-15-96116, and by INTAS-265. 
20 years $[2-4,7,10]$. This is, partially, due to the fact that this property allows one to consider the operator $\mathcal{S}$ in the set of all continuous inputs $u(\cdot)$ which is more natural for the investigation of stability with respect to small perturbations.

The case when the reflection cone $\mathcal{R}(z)$ coincides with the outward normal cone to $Z$ at each point $z \in Z$, like for instance in stress-strain laws of elastoplasticity, where the normality rule follows from the maximal dissipation condition, constitutes the important class of polyhedral Skorokhod problems with normal reflection. The corresponding Skorokhod map then represents the constitutive operator of the Prager linear kinematic hardening model and is called multi-dimensional play operator. Its Lipschitz continuity with respect to the sup-norm was first proved in [10] (see also [7] where this theorem is reproduced), then (by a different method) in [3, 4]. Recently, in [8], a recurrent upper bound for the Lipschitz constant has been found.

The general situation of oblique reflection arises in various models of human activity, and below in Section 8 we show a typical example from queuing theory. Sufficient conditions for the Lipschitz continuity were formulated in $[3,4]$ in terms of existence of a special convex set $B \in X$ with $0 \in \operatorname{Int} B$. Conditions of existence of solution can also be found in $[3,4]$; however, they are different from the sufficient conditions of Lipschitz continuity and require additional assumptions on the reflection directions.

In all applications, the question of Lipschitz continuity of the input-output operator is substantial for the stability of numerical computations. An explicit knowledge of the Lipschitz constant is useful in particular for estimating the discretization error and the efficiency of the algorithm.

The analysis of the Skorokhod problem in this paper is based on the concept of $\ell$ paracontractivity introduced in [6]. This is a special stability property of the associated projection system of linear operators of oblique projection on hyperplanes parallel to the faces of $Z$ along the reflection directions (see Section 3). We first prove that $\ell-$ paracontractivity alone is sufficient for the existence of an absolutely continuous output $x(t)$ for every absolutely continuous input $u(t)$ and every initial condition. If, in addition, the associated projection system is transversal, that is, no reflection direction at a point $z$ is orthogonal to all normal directions at $z$, then the Skorokhod map is of Lipschitz type in the space $W^{1,1}(0, T ; X)$ as well as in the space $C([0, T] ; X)$ of continuous functions. If moreover $Z$ has non-empty interior, then, for every continuous function $u$, the function $\xi=\mathcal{S}\left[x_{0}, u\right]$ has bounded variation.

An important property of $\ell$-paracontracting sets of oblique projections is their robustness with respect to small shifts of reflection vectors for fixed normal directions. This property implies the Lipschitz continuity of Skorokhod problems under the transversality constraint whenever the reflection vectors are close to normal ones. On the other hand, it does not yield an explicit upper bound for the Lipschitz constant of a deviated Skorokhod problem. We obtain independently such an upper bound by a modified method of Lyapunov functions (cf. [8]).

The paper is organized as follows. In Section 1 we state the Skorokhod problem in the space of continuous functions. Section 2 is devoted to a survey of basic properties of oblique projections. In Section 3 we prove that the $\ell$-paracontractivity ensures the existence of a solution for each initial condition. In Section 4 we establish a Lipschitztype estimate for the sup-norm. Section 5 contains the main result which consists in 
proving that $\ell$-paracontractivity and transversality imply the Lipschitz continuity of the Skorokhod map in both spaces $W^{1,1}(0, T ; X)$ and $C([0, T] ; X)$. In Section 6 we derive an estimate for the total variation of the output, and an upper bound for the Lipschitz constant is derived in Section 7. We conclude the paper with an example from queuing theory in Section 8.

\section{The Skorokhod problem}

Let $X$ be a Hilbert space endowed with a scalar product $\langle\cdot, \cdot\rangle$ and with the norm $|\cdot|=\langle\cdot, \cdot\rangle^{\frac{1}{2}}$. We consider a polyhedral set $Z \subset X$ defined in terms of a system $n_{1}, \ldots, n_{p}$ of unit outward normal vectors as the intersection of half-spaces $H_{j} \quad(j=1, \ldots, p)$ by the formula

$$
Z=\bigcap_{j \in J} H_{j}, \quad H_{j}=\left\{z \in X:\left\langle z, n_{j}\right\rangle \leq \beta_{j} \text { for } j \in J\right\}, \quad J=\{1, \ldots, p\}
$$

where $\beta_{j} \geq 0$ are given real numbers. We associate with $Z$ a system $\left\{r_{1}, \ldots, r_{p}\right\}$ of unit vectors called reflection vectors. For $z \in Z$ we denote by

$$
\tilde{J}(z)=\left\{j \in J:\left\langle z, n_{j}\right\rangle=\beta_{j}\right\}
$$

the set of indices corresponding to 'active' constraints at the point $z$. The set-valued mapping $\tilde{J}: Z \rightarrow 2^{J}$ is upper semicontinuous in the sense that for all $z \in Z$ there exists $\varepsilon>0$ such that

$$
\left|z^{\prime}-z\right|<\varepsilon \quad \Longrightarrow \quad \tilde{J}\left(z^{\prime}\right) \subset \tilde{J}(z)
$$

Indeed, it suffices to put

$$
\varepsilon=\min \left\{\beta_{j}-\left\langle z, n_{j}\right\rangle: j \in J \backslash \tilde{J}(z)\right\} .
$$

For a function $w:[0, T] \rightarrow Z$ and any subset $A \subset[0, T]$ we put

$$
\tilde{J}_{A}(w)=\bigcup_{t \in A} \tilde{J}(w(t))
$$

For any subset $J^{\prime} \subset J$ we denote by $\mathcal{C}\left(J^{\prime}\right)$ the convex cone generated by vectors $r_{j}$ with indices from $J^{\prime}$, that is

$$
\mathcal{C}\left(J^{\prime}\right)=\left\{y=\sum_{j \in J^{\prime}} \alpha_{j} r_{j} \mid \alpha_{j} \geq 0 \text { for } j \in J^{\prime}\right\}
$$

and for $z \in Z$ we call the set

$$
\mathcal{R}(z)=\mathcal{C}(\tilde{J}(z))
$$

the reflection cone at the point $z$. Similarly, for a function $w:[0, T] \rightarrow Z$ and any set $A \subset[0, T]$ we define

$$
\mathcal{R}_{A}(w)=\mathcal{C}\left(\tilde{J}_{A}(w)\right)
$$

As an immediate consequence of (1.3), we see that for every $w \in C([0, T] ; Z)$ and every compact set $A \subset[0, T]$ there exists $\varepsilon>0$ such that for each $\tilde{w} \in C([0, T] ; Z)$ the implication

$$
|w-\tilde{w}|_{A}<\varepsilon \quad \Longrightarrow \quad \mathcal{R}_{A}(\tilde{w}) \subset \mathcal{R}_{A}(w)
$$

holds where for $v \in C([0, T] ; X)$ we put $|v|_{A}=\max _{t \in A}|v(t)|$.

We state the Skorokhod problem in the framework of continuous functions as follows: 
Definition 1.1. Let $u \in C([0, T] ; X)$ be a given function. A pair of functions $\xi, x \in C([0, T] ; X)$ is said to be a solution to the Skorokhod problem with characteristic $Z$ given by (1.1) and with reflection vectors $r_{1}, \ldots, r_{p}$, if

$$
\left.\begin{array}{rlrl}
x(t)+\xi(t) & =u(t) & & \text { for every } t \in[0, T] \\
x(t) & \in Z & & \text { for every } t \in[0, T] \\
\xi\left(t_{2}\right)-\xi\left(t_{1}\right) & \in \mathcal{R}_{\left[t_{1}, t_{2}\right]}(x) & & \text { for every } 0 \leq t_{1}<t_{2} \leq T
\end{array}\right\} .
$$

The alternative formulation given in $[3,4]$ includes also discontinuous inputs and outputs. The restriction to continuous functions enables us to make the geometrical ideas more clear and the proofs more transparent. Due to (1.3), we see that whenever the derivatives $\dot{u}(t), \dot{x}(t), \dot{\xi}(t)$ exist for some $t$, the third condition in (1.7) yields

$$
\dot{\xi}(t) \in \mathcal{R}(x(t)) .
$$

In other words, the vector $\dot{u}(t)$ is decomposed into a tangential component $\dot{x}(t)$ and a reflection component $\dot{\xi}(t)$. The problem has been studied in detail in the case of normal reflection, that is, $n_{j}=r_{j}$ for every $j \in J$, and a survey of results can be found in [2]. In fact, the Skorokhod problem can then be stated as an evolution variational inequality in a Hilbert space which makes it accessible to classical analytical methods. Here, we are particularly interested in the case of oblique reflection, where no a priori assumption is made on the relationship between $n_{j}$ and $r_{j}$.

We immediately see, however, that a necessary condition for the well-posedness of the Skorokhod problem reads

$$
\left\langle r_{j}, n_{j}\right\rangle>0
$$

whenever the $j$-th constraint is non-degenerate, that is, if there exists $x_{j} \in Z$ such that $\tilde{J}\left(x_{j}\right)=\{j\}$. Indeed, if $\left\langle r_{j}, n_{j}\right\rangle \leq 0$, then taking $x(0)=x_{j}$ and $\dot{u}(t) \equiv n_{j}$ in $[0, T]$, we conclude from the convexity of $Z$ and from (1.6) that $\left\langle x(t)-x(0), n_{j}\right\rangle \leq 0$ and $\left\langle\xi(t)-\xi(0), n_{j}\right\rangle \leq 0$ for small $t>0$, which is a contradiction.

Put

$$
Y=\operatorname{span}\left\{n_{1}, \ldots, n_{p} ; r_{1}, \ldots, r_{p}\right\}
$$

and let $Y^{\perp}$ be the orthogonal complement of $Y$ in $X$. For every functions $u, x, \xi \in$ $C([0, T] ; X)$ satisfying $(1.7)$ and an arbitrary $w \in C\left(0, T ; Y^{\perp}\right)$, the functions $\tilde{u}=u+$ $w, \tilde{x}=x+w, \tilde{\xi}=\xi$ also satisfy (1.7). We can therefore restrict our considerations to the (finite-dimensional) space $Y$ instead of $X$.

This motivates the following hypothesis which is assumed to be valid in all what follows:

Hypothesis 1.2. $X=\operatorname{span}\left\{n_{1}, \ldots, n_{p} ; r_{1}, \ldots, r_{p}\right\}$ and (1.9) holds for every $j \in J$.

If the solution to the Skorokhod problem with a given initial condition $x(0)=x_{0} \in Z$ is unique, we define the Skorokhod map $\mathcal{S}: Z \times C([0, T] ; X) \rightarrow C([0, T] ; X)$ by the formula

$$
\mathcal{S}\left[x_{0}, u\right]=\xi .
$$

By construction, the mapping $\mathcal{S}$ is causal and rate-independent, hence it belongs to the class of hysteresis operators. 


\section{Oblique projections}

For $j \in J$, let $Q_{j}$ be the projection onto $\operatorname{span}\left\{r_{j}\right\}$ orthogonal to $n_{j}$, that is,

$$
Q_{j} x=\frac{\left\langle x, n_{j}\right\rangle}{\left\langle r_{j}, n_{j}\right\rangle} r_{j} \quad \text { for } x \in X .
$$

The family $\mathcal{Q}$ of complementary projections $\left\{\left(I-Q_{j}\right) \mid j \in J\right\}$, where $I: X \rightarrow X$ is the identity mapping, is called the associated projection system of the Skorokhod problem.

Let us introduce the following basic definition (cf. [6]).

Definition 2.1. Let Hypothesis 1.2 hold. The system $\mathcal{Q}$ is said to be $\ell$-paracontracting if there exists a norm in $X$ denoted by $\|\cdot\|$ such that for every $x \in X$ and every $j \in J$ we have

$$
\|x\| \geq\left\|\left(I-Q_{j}\right) x\right\|+\left|Q_{j} x\right| .
$$

In the case of the Skorokhod problem with normal reflection, such a norm can be constructed explicitly (see $[1,2,9]$ ).

The following result shows that the $\ell$-paracontracting property is robust with respect to small shifts of the reflection vectors. In particular, it remains valid if the reflection directions are sufficiently close to the normal ones.

Lemma 2.2. Let the system $\mathcal{Q}$ be $\ell$-paracontracting and let $\left\{r_{1}^{\prime}, \ldots, r_{p}^{\prime}\right\}$ be a set of unit vectors such that for every $j \in J$ we have

$$
\left\langle n_{j}, r_{j}^{\prime}\right\rangle>0, \quad\left|\frac{r_{j}}{\left\langle n_{j}, r_{j}\right\rangle}-\frac{r_{j}^{\prime}}{\left\langle n_{j}, r_{j}^{\prime}\right\rangle}\right|+\left\|\frac{r_{j}}{\left\langle n_{j}, r_{j}\right\rangle}-\frac{r_{j}^{\prime}}{\left\langle n_{j}, r_{j}^{\prime}\right\rangle}\right\|<\frac{1}{\left\langle n_{j}, r_{j}^{\prime}\right\rangle} .
$$

Then the system $\mathcal{Q}^{\prime}$ of projections $I-Q_{j}^{\prime}$, where the vectors $r_{j}$ are replaced with $r_{j}^{\prime}$, is also an $\ell$-paracontracting system.

Proof. Put

$$
\delta=\max _{j \in J}\left\{\left\langle n_{j}, r_{j}^{\prime}\right\rangle\left(\left|\frac{r_{j}}{\left\langle n_{j}, r_{j}\right\rangle}-\frac{r_{j}^{\prime}}{\left\langle n_{j}, r_{j}^{\prime}\right\rangle}\right|+\left\|\frac{r_{j}}{\left\langle n_{j}, r_{j}\right\rangle}-\frac{r_{j}^{\prime}}{\left\langle n_{j}, r_{j}^{\prime}\right\rangle}\right\|\right)\right\}<1 .
$$

For every $j \in J$ and $x \in X$ we then have

$$
\begin{aligned}
\left\|\left(I-Q_{j}^{\prime}\right) x\right\| & \leq\left\|\left(I-Q_{j}\right) x\right\|+\left\|\left(Q_{j}^{\prime}-Q_{j}\right) x\right\| \\
& \leq\|x\|-\left|Q_{j}^{\prime} x\right|+\left|\left(Q_{j}^{\prime}-Q_{j}\right) x\right|+\left\|\left(Q_{j}^{\prime}-Q_{j}\right) x\right\| \\
& \leq\|x\|-\left|Q_{j}^{\prime} x\right|+\left|\left\langle x, n_{j}\right\rangle\right|\left(\left|\frac{r_{j}}{\left\langle n_{j}, r_{j}\right\rangle}-\frac{r_{j}^{\prime}}{\left\langle n_{j}, r_{j}^{\prime}\right\rangle}\right|+\left\|\frac{r_{j}}{\left\langle n_{j}, r_{j}\right\rangle}-\frac{r_{j}^{\prime}}{\left\langle n_{j}, r_{j}^{\prime}\right\rangle}\right\|\right) \\
& \leq\|x\|-\left|Q_{j}^{\prime} x\right|+\delta\left|\frac{\left\langle x, n_{j}\right\rangle}{\left\langle n_{j}, r_{j}^{\prime}\right\rangle}\right| \\
& =\|x\|-(1-\delta)\left|Q_{j}^{\prime} x\right| .
\end{aligned}
$$

Dividing this inequality by $1-\delta$, we see that the assertion holds with respect to the norm $\|\cdot\|^{\prime}=\frac{1}{1-\delta}\|\cdot\|$ 
We have the following easy consequence of Definition 2.1.

Lemma 2.3. If $\mathcal{Q}$ is $\ell$-paracontracting, then

$$
\|x\| \geq\left\|\left(I-\gamma Q_{j}\right) x\right\|+\gamma\left|Q_{j} x\right|
$$

for every $j \in J, x \in X$ and $0 \leq \gamma \leq 1$.

Proof. Multiplying (2.2) by $\gamma$ and using the triangle inequality, we get

$$
\gamma\|x\| \geq\left\|\gamma\left(I-Q_{j}\right) x\right\|+\gamma\left|Q_{j} x\right| \geq\left\|\left(I-\gamma Q_{j}\right) x\right\|-(1-\gamma)\|x\|+\gamma\left|Q_{j} x\right|
$$

and (2.3) follows easily

Let us define nonlinear operators of oblique projection onto half-spaces $H_{j} \quad(j \in J)$ as

$$
\pi_{j}(x)= \begin{cases}x & \text { if }\left\langle x, n_{j}\right\rangle \leq \beta_{j} \\ \left(I-Q_{j}\right) x+\beta_{j} Q_{j} n_{j} & \text { if }\left\langle x, n_{j}\right\rangle>\beta_{j}\end{cases}
$$

We will need the following two properties of the operators $\pi_{j}$.

Proposition 2.4. Let $\mathcal{Q}$ be $\ell$-paracontracting. Then for each $j \in J$ the following inequalities hold:

(i) $\left|\pi_{j}(x)-x\right| \leq\|x-z\|-\left\|\pi_{j}(x)-z\right\|$ for all $x \in X$ and all $z \in H_{j}$.

(ii) $\left\|\pi_{j}\left(x_{1}\right)-\pi_{j}\left(x_{2}\right)\right\| \leq\left\|x_{1}-x_{2}\right\|-\left|\left(x_{1}-\pi_{j}\left(x_{1}\right)\right)-\left(x_{2}-\pi_{j}\left(x_{2}\right)\right)\right|$ for all $x_{1}, x_{2} \in X$.

Proof. (i) Let us denote $v=x-z$ and $w=\pi_{j}(x)-z$. Then

$$
w=\left(I-\gamma Q_{j}\right) v \quad \text { where } \gamma= \begin{cases}0 & \text { if }\left\langle x, n_{j}\right\rangle \leq \beta_{j} \\ \frac{\left\langle x, n_{j}\right\rangle-\beta_{j}}{\left\langle x, n_{j}\right\rangle-\left\langle z, n_{j}\right\rangle} & \text { if }\left\langle x, n_{j}\right\rangle>\beta_{j}\end{cases}
$$

We have $0 \leq \gamma \leq 1$ because $\left\langle z, n_{j}\right\rangle \leq \beta_{j}$. Hence the assertion follows from Lemma 2.3.

(ii) If $\left\langle x_{i}, n_{j}\right\rangle \leq \beta_{j}$ for one or both of $x_{1}$ and $x_{2}$, it suffices to use assertion (i). Otherwise $\pi_{j}\left(x_{1}\right)-\pi_{j}\left(x_{2}\right)=\left(I-Q_{j}\right)\left(x_{1}-x_{2}\right)$ and the statement follows directly from $(2.2)$

We further define a mapping $\pi: X \rightarrow Z$ called quasiprojection such that for every $x \in X$ close to a point $z \in Z$ the difference $x-\pi(x)$ lies in the reflection cone of $z$ (a precise formulation will be given in Proposition 2.6 below).

We take a specific sequence $\left\{j_{k}\right\}_{k \geq 0}$ of indices from $J$, namely

$$
j_{k}=k[\bmod p]+1 \quad\left(k \in \mathbb{N}_{0}\right)
$$

and for a given $x \in X$ we define recursively the sequence

$$
\left.\begin{array}{rl}
y_{0} & =x \\
y_{k+1} & =\pi_{j_{k}}\left(y_{k}\right) \quad\left(k \in \mathbb{N}_{0}\right)
\end{array}\right\} .
$$


By construction, $y_{k+1} \in H_{j_{k}}$ for every $k \in \mathbb{N}_{0}$. Moreover, from Proposition 2.4 we get

$$
\sum_{k=0}^{\infty}\left|y_{k+1}-y_{k}\right| \leq\|x-z\| \quad(z \in Z)
$$

Hence the sequence $\left\{y_{k}\right\}$ is convergent and we define the quasiprojection operator $\pi$ : $X \rightarrow X$ by

$$
\pi(x)=\lim _{k \rightarrow \infty} y_{k} \quad \text { for } x \in X .
$$

From the construction $\pi(x) \in Z$ follows.

We now list further properties of $\pi$.

Proposition 2.5. Let $\mathcal{Q}$ be $\ell$-paracontracting. Then for every $x \in X$ we have

(i) $\|\pi(x)-z\| \leq\|x-z\|-|x-\pi(x)|$ for all $z \in Z$

(ii) $\|x-\pi(x)\| \leq 2 \min _{z \in Z}\|x-z\|$

(iii) $\left\|\pi\left(x_{1}\right)-\pi\left(x_{2}\right)\right\| \leq\left\|x_{1}-x_{2}\right\|-\left|\left(x_{1}-\pi\left(x_{1}\right)\right)-\left(x_{2}-\pi\left(x_{2}\right)\right)\right|$ for all $x_{1}, x_{2} \in X$.

Proof. (i) Let $\left\{y_{k}\right\}$ be the sequence (2.6). By Proposition 2.4/(i),

$$
\left|y_{k+1}-y_{k}\right| \leq\left\|y_{k}-z\right\|-\left\|y_{k+1}-z\right\|
$$

for every $k$. Summing up over $k \in \mathbb{N}_{0}$ we obtain the assertion.

(ii) Let $z^{*} \in Z$ be such that $\left\|x-z^{*}\right\|=\min _{z \in Z}\|x-z\|$. From (2.8) we obtain $\left\|y_{k}-z^{*}\right\| \leq\left\|y_{0}-z^{*}\right\|$, hence

$$
\left\|x-y_{k}\right\| \leq\left\|x-z^{*}\right\|+\left\|y_{k}-z^{*}\right\| \leq 2\left\|x-z^{*}\right\|
$$

and assertion (ii) follows.

(iii) Let $\left\{y_{k}^{(i)}\right\}$ for $i=1,2$ be sequences (2.6) with initial conditions $y_{0}^{(i)}=x_{i}$. By Proposition 2.4/(ii), for all $k$ we have

$$
\left\|y_{k+1}^{(1)}-y_{k+1}^{(2)}\right\| \leq\left\|y_{k}^{(1)}-y_{k}^{(2)}\right\|-\left|\left(y_{k}^{(1)}-y_{k+1}^{(1)}\right)-\left(y_{k}^{(2)}-y_{k+1}^{(2)}\right)\right|
$$

and, analogously to assertion (i), a summation argument completes the proof

The following property of $\pi$ plays a substantial role in our argument.

Proposition 2.6. Let $\mathcal{Q}$ be $\ell$-paracontracting. Let $z \in Z$ be given and let $\varepsilon>0$ be such that the implication

$$
\|x-z\|<\varepsilon \quad \Longrightarrow \quad\left\langle x, n_{j}\right\rangle<\beta_{j} \forall j \in J \backslash \tilde{J}(z)
$$

holds for every $x \in X$. Then

$$
x-\pi(x) \in \mathcal{R}(z) \quad \forall x \in X \text { with }\|x-z\|<\varepsilon
$$

where $\mathcal{R}(z)$ is the reflection cone defined by (1.4). 

hence

Proof. Let $\left\{y_{k}\right\}$ be sequence (2.6). By (2.9), $\left\|y_{k}-z\right\| \leq\|x-z\|<\varepsilon$ for every $k$,

$$
\left\langle y_{k}, n_{j}\right\rangle<\beta_{j} \quad \forall j \in J \backslash \tilde{J}(z) .
$$

On the other hand,

$$
x-\pi(x)=\sum_{k=0}^{\infty}\left(y_{k}-y_{k+1}\right)=\sum_{k \in K} Q_{j_{k}}\left(y_{k}-\beta_{j_{k}} n_{j_{k}}\right)=\sum_{k \in K} \frac{\left\langle y_{k}, n_{j_{k}}\right\rangle-\beta_{j_{k}}}{\left\langle r_{j_{k}}, n_{j_{k}}\right\rangle} r_{j_{k}}
$$

where $K=\left\{k:\left\langle y_{k}, n_{j_{k}}\right\rangle>\beta_{j_{k}}\right\}$. Therefore, by $(2.10), j_{k} \in \tilde{J}(z)$ for every $k \in K$, and from (2.11) we conclude that there exist coefficients $\alpha_{j} \geq 0$ such that

$$
x-\pi(x)=\sum_{j \in \tilde{J}(z)} \alpha_{j} r_{j}
$$

which we wanted to prove

\section{Skorokhod problem in $W^{1,1}(0, T ; X)$}

We first solve the Skorokhod problem for absolutely continuous input functions $u$. Keeping the notation from Section 2, we construct a solution by time-discrete approximation.

With any given input sequence (finite or infinite) $\left\{u_{0}, u_{1}, \ldots\right\}$ and initial condition $x_{0} \in Z$ we associate output sequences $\left\{x_{0}, x_{1}, \ldots\right\}$ and $\left\{\xi_{0}, \xi_{1}, \ldots\right\}$ by the recurrent formulas

$$
\left.\begin{array}{rl}
x_{i+1} & =\pi\left(x_{i}+u_{i+1}-u_{i}\right) \\
\xi_{i} & =u_{i}-x_{i}
\end{array}\right\} \quad\left(i \in \mathbb{N}_{0}\right)
$$

where $\pi$ is the quasiprojection operator (2.7). For every $i \geq 1$ we have in particular $x_{i} \in Z$ and

$$
\xi_{i}-\xi_{i-1}=\left(x_{i-1}+u_{i}-u_{i-1}\right)-\pi\left(x_{i-1}+u_{i}-u_{i-1}\right),
$$

hence Proposition 2.5 yields

$$
\left|\xi_{i}-\xi_{i-1}\right| \leq\left\|x_{i-1}+u_{i}-u_{i-1}-z\right\|-\left\|x_{i}-z\right\| \quad(z \in Z) .
$$

Let two input sequences $\left\{u_{i}^{(j)}\right\} \quad(j=1,2)$ be given. We denote by $\left\{x_{i}^{(j)}\right\},\left\{\xi_{i}^{(j)}\right\}$ the corresponding output sequences and by $\left\{\bar{u}_{i}\right\},\left\{\bar{x}_{i}\right\},\left\{\bar{\xi}_{i}\right\}$ the differences $\bar{u}_{i}=u_{i}^{(2)}-u_{i}^{(1)}, \bar{x}_{i}=$ $x_{i}^{(2)}-x_{i}^{(1)}, \bar{\xi}_{i}=\xi_{i}^{(2)}-\xi_{i}^{(1)}$. From Proposition 2.5/(iii) we then obtain

$$
\left|\bar{\xi}_{i}-\bar{\xi}_{i-1}\right| \leq\left\|\bar{x}_{i-1}+\bar{u}_{i}-\bar{u}_{i-1}\right\|-\left\|\bar{x}_{i}\right\| .
$$

The existence result can be stated as follows. 
Theorem 3.1. Let $\mathcal{Q}$ be an $\ell$-paracontracting system, and let $u \in W^{1,1}(0, T ; X)$ and $x_{0} \in Z$ be given. Then there exist functions $x, \xi \in W^{1,1}(0, T ; X)$ satisfying the conditions of Definition 1.1 and $x(0)=x_{0}$.

Proof. For a given $n \in \mathbb{N}$, we divide the interval $[0, T]$ into an equidistant partition

$$
0=t_{0}^{(n)}<t_{1}^{(n)}<\ldots<t_{n}^{(n)}=T, \quad t_{i}^{(n)}=\frac{i}{n} T \quad(i=0, \ldots, n)
$$

and put, keeping $n$ fixed for the moment,

$$
u_{i}=u\left(t_{i}^{(n)}\right) \quad(i=0, \ldots, n) .
$$

Let an initial condition $x_{0}$ be given. We define $x_{i}$ for $i=1, \ldots, n$ by formula (3.1), and for $t \in\left[t_{i-1}^{(n)}, t_{i}^{(n)}\right)$ we put

$$
\left.\begin{array}{l}
u^{(n)}(t)=u_{i-1}+\frac{n}{T}\left(t-t_{i-1}^{(n)}\right)\left(u_{i}-u_{i-1}\right) \\
x^{(n)}(t)=x_{i-1}+\frac{n}{T}\left(t-t_{i-1}^{(n)}\right)\left(x_{i}-x_{i-1}\right)
\end{array}\right\} .
$$

As a consequence of $(3.3)$ where we put $z=x_{i-1}$, for every $i=1, \ldots, n$ the inequality

$$
\left\|x_{i}-x_{i-1}\right\| \leq\left\|u_{i}-u_{i-1}\right\|
$$

holds. The sequence $\left\{x^{(n)}\right\}$ is thus equibounded in $C([0, T] ; X)$ and $\left\{\dot{x}^{(n)}\right\}$ is equiintegrable in $L^{1}(0, T ; X), x^{(n)}(t) \in Z$ for every $t \in[0, T]$. There exists therefore $x \in W^{1,1}(0, T ; X)$ such that $x(t) \in Z$ for every $t \in[0, T]$ and $x(0)=x_{0}$, and a subsequence of $\left\{x^{(n)}\right\}$ (still indexed by $(n)$ ) such that $x^{(n)} \rightarrow x$ uniformly in $C([0, T] ; X)$ and $\dot{x}^{(n)} \rightarrow \dot{x}$ in $L^{1}(0, T ; X)$ weakly as $n \rightarrow \infty$.

It remains to prove that the function $\xi(t)=u(t)-x(t)$ satisfies for a.e. $t \in(0, T)$ the condition

$$
\dot{\xi}(t) \in \mathcal{R}(x(t)) .
$$

Let $t \in(0, T)$ be a Lebesgue point of both $u$ and $x$, and let $\varepsilon>0$ be chosen according to (1.3) in such a way that the implication

$$
\|x(t)-\hat{x}\|<\varepsilon \quad \Longrightarrow \quad\left\langle\hat{x}, n_{j}\right\rangle<\beta_{j} \forall j \in J \backslash \tilde{J}(x(t))
$$

holds for every $\hat{x} \in X$. We fix $n_{0} \in \mathbb{N}$ and $\delta>0$ such that

$$
\begin{aligned}
\max _{\tau \in[0, T]}\left\|x^{(n)}(\tau)-x(\tau)\right\|<\frac{1}{3} \varepsilon & \text { for } n \geq n_{0} \\
\|x(t)-x(\tau)\|<\frac{1}{3} \varepsilon & \text { for } \tau \in(t-\delta, t+\delta) \\
\|u(\sigma)-u(\tau)\|<\frac{1}{3} \varepsilon & \text { for } \sigma, \tau \in(t-\delta, t+\delta) .
\end{aligned}
$$

Let now $n \geq n_{0}$ and $i \in\{1, \ldots, n\}$ be such that $t_{i-1}^{(n)}, t_{i}^{(n)} \in(t-\delta, t+\delta)$, and for $\tau \in(t-\delta, t+\delta)$ put $\xi^{(n)}(\tau)=u^{(n)}(\tau)-x^{(n)}(\tau)$. Then 


$$
\xi^{(n)}\left(t_{i}^{(n)}\right)-\xi^{(n)}\left(t_{i-1}^{(n)}\right)=\left(x_{i-1}+u_{i}-u_{i-1}\right)-\pi\left(x_{i-1}+u_{i}-u_{i-1}\right) .
$$

According to $(3.10)-(3.12)$, the point $\hat{x}=x_{i-1}+u_{i}-u_{i-1}$ satisfies the inequality

$$
\|\hat{x}-x(t)\| \leq\left\|x^{(n)}\left(t_{i-1}^{(n)}\right)-x(t)\right\|+\left\|u\left(t_{i}^{(n)}\right)-u\left(t_{i-1}^{(n)}\right)\right\|<\varepsilon
$$

and from (3.9) and Proposition 2.6

$$
\xi^{(n)}\left(t_{i}^{(n)}\right)-\xi^{(n)}\left(t_{i-1}^{(n)}\right) \in \mathcal{R}(x(t))
$$

follows. Since the functions $\xi^{(n)}$ are piecewise linear, for large $n$ we have

$$
\xi^{(n)}\left(t_{2}\right)-\xi^{(n)}\left(t_{1}\right) \in \mathcal{R}(x(t))
$$

for every $t-\delta<t_{1} \leq t \leq t_{2}<t+\delta$, and passing to the limit we obtain (3.8). The proof is complete

Remark 3.2. If $u_{1}, u_{2} \in W^{1,1}(0, T ; X)$ are two input functions, then from (3.4) it follows for the piecewise linear approximations that for $t \in\left(t_{i-1}^{(n)}, t_{i}^{(n)}\right)$ we have

$$
\left|\dot{\xi}_{2}^{(n)}(t)-\dot{\xi}_{1}^{(n)}(t)\right|+\frac{n}{T}\left(\bar{x}_{i}^{(n)}-\bar{x}_{i-1}^{(n)}\right) \leq\left\|\dot{u}_{2}^{(n)}(t)-\dot{u}_{1}^{(n)}(t)\right\|
$$

where $\bar{x}_{i}^{(n)}=\left\|x_{2}^{(n)}\left(t_{i}^{(n)}\right)-x_{1}^{(n)}\left(t_{i}^{(n)}\right)\right\|$.

Let $0<a<b<T$ be arbitrarily chosen. For $n$ sufficiently large we find indices $1<j<k<n$ such that $t_{j-2}^{(n)}<a \leq t_{j-1}^{(n)}$ and $t_{k}^{(n)} \leq b<t_{k+1}^{(n)}$. Integrating (3.13) we obtain

$$
\begin{aligned}
\int_{a}^{b}\left|\dot{\xi}_{2}^{(n)}(t)-\dot{\xi}_{1}^{(n)}(t)\right| d t & +\left(c_{k} \bar{x}_{k+1}^{(n)}+\left(1-c_{k}\right) \bar{x}_{k}^{(n)}\right)-\left(d_{j} \bar{x}_{j-2}^{(n)}+\left(1-d_{j}\right) \bar{x}_{j-1}^{(n)}\right) \\
& \leq \int_{a}^{b}\left\|\dot{u}_{2}^{(n)}(t)-\dot{u}_{1}^{(n)}(t)\right\| d t
\end{aligned}
$$

where $c_{k}=\frac{\left(b-t_{k}^{(n)}\right) n}{T}$ and $d_{j}=\frac{\left(t_{j-1}^{(n)}-a\right) n}{T}$. The sequences $\left\{u_{1}^{(n)}\right\},\left\{u_{2}^{(n)}\right\}$ converge strongly in $W^{1,1}(0, T ; X)$ and $\left\{\dot{\xi}_{1}^{(n)}\right\},\left\{\dot{\xi}_{2}^{(n)}\right\}$ converge weakly in $L^{1}(0, T ; X)$. Passing to the limit as $n \rightarrow \infty$ in (3.14) we thus obtain

$$
\begin{aligned}
\int_{a}^{b}\left|\dot{\xi}_{2}(t)-\dot{\xi}_{1}(t)\right| d t & \leq \liminf _{n \rightarrow \infty} \int_{a}^{b}\left|\dot{\xi}_{2}^{(n)}(t)-\dot{\xi}_{1}^{(n)}(t)\right| d t \\
& \leq\left\|x_{2}(a)-x_{1}(a)\right\|-\left\|x_{2}(b)-x_{1}(b)\right\|+\int_{a}^{b}\left\|\dot{u}_{2}(t)-\dot{u}_{1}(t)\right\| d t
\end{aligned}
$$

Since $a$ and $b$ have been arbitrary, we can write the above inequality in differential form

$$
\left|\dot{\xi}_{2}(t)-\dot{\xi}_{1}(t)\right|+\frac{d}{d t}\left\|x_{2}(t)-x_{1}(t)\right\| \leq\left\|\dot{u}_{2}(t)-\dot{u}_{1}(t)\right\| \quad \text { a.e. }
$$


which is the same as in the normal reflection case (see [1]).

We cannot conclude for the moment that the solution to the Skorokhod problem is unique in $W^{1,1}(0, T ; X)$ (see Example 3.3 below); we only made sure that solutions which can be constructed as discrete limits are unique. The uniqueness and Lipschitz continuity in $W^{1,1}(0, T ; X)$ will be obtained under an additional assumption below in Theorem 5.8.

Example 3.3. Let $\left\{e_{1}, e_{2}\right\}$ be an orthonormal basis in $X=\mathbb{R}^{2}$. We consider the set $Z=\left\{x \in X:\left\langle x, e_{1}\right\rangle=0\right\}$. This corresponds to the choice $n_{1}=-n_{2}=e_{1}$ and

$\beta_{1}=\beta_{2}=0$ in (1.1). We choose the reflection vectors $r_{1}=\frac{e_{2}+e_{1}}{\sqrt{2}}$ and $r_{2}=\frac{e_{2}-e_{1}}{\sqrt{2}}$. Then the system $\mathcal{Q}$ is $\ell$-paracontracting with the norm

$$
\|x\|=(1+\sqrt{2})\left|\left\langle x, e_{1}\right\rangle\right|+\left|\left\langle x, e_{2}\right\rangle\right|
$$

For the input function $u(t) \equiv 0$, all functions of the form $\xi(t)=\lambda(t) e_{2}$ and $x(t)=$ $-\lambda(t) e_{2}$ with a non-decreasing function $\lambda$ such that $\lambda(0)=0$ are solutions of the Skorokhod problem (1.7) with initial condition $x(0)=0$. However, the time discretization method converges to the trivial solution $\xi=x \equiv 0$.

\section{Uniqueness and Lipschitz continuity in $C([0, T] ; X)$}

Sufficient conditions for Lipschitz continuity of the Skorokhod map with respect to the norm $|\cdot|_{[0, T]}$ of uniform convergence were given in $[3,4]$ in terms of existence of a special bounded set $B \subset X$ (condition $(\mathcal{B})$ in Theorem 4.1 below, with the additional requirement $0 \in \operatorname{Int}(B))$. We now study this problem in more detail and summarize our results in Theorem 4.9 at the end of this section.

We first derive some geometrical properties of the associated projection system.

Theorem 4.1. Let Hypothesis 1.2 hold, let $Q_{j}(j \in J)$ be the projections defined by (2.1) and let $B \subset X$ be a closed convex set with $0 \in B$. Then the following two conditions are equivalent:

$(\mathcal{A})$ For all $x \in B$ and all $j \in J, w:=\left(I-Q_{j}\right) x \pm Q_{j} n_{j} \in B$ where $I$ is the identity operator.

$(\mathcal{B})$ For all $x \in B$, all $y \in \mathcal{N}_{B}(x)$ and all $j \in J,\left|\left\langle x, n_{j}\right\rangle\right|<1$ implies $\left\langle y, r_{j}\right\rangle=0$ where $\mathcal{N}_{B}(x)$ denotes the outward normal cone to $B$ at the point $x$.

Notation 4.2. In the sequel, by a $\mathcal{Q}$-invariant set we understand any convex closed set $B$ containing the origin and satisfying condition $(\mathcal{A})$.

Proof of Theorem 4.1.

$(\mathcal{A}) \Rightarrow(\mathcal{B})$ : By definition, for every $x \in B$ and every $y \in \mathcal{N}_{B}(x),\langle y, x-w\rangle \geq 0$ for all $w \in B$. Assuming condition $(\mathcal{A})$, we may put $w=\left(I-Q_{j}\right) x \pm Q_{j} n_{j}$ and obtain

$$
0 \leq\left\langle y, Q_{j}\left(x \mp n_{j}\right)\right\rangle=\left\langle y, r_{j}\right\rangle \frac{\left\langle x, n_{j}\right\rangle \mp 1}{\left\langle n_{j}, r_{j}\right\rangle} .
$$


If $\left|\left\langle x, n_{j}\right\rangle\right|<1$ for some $j \in J$, the above inequality immediately yields $\left\langle y, r_{j}\right\rangle=0$ and $(\mathcal{B})$ follows.

$(\mathcal{B}) \Rightarrow(\mathcal{A})$ : Let $x \in B$ and $j \in J$ be given and let $A$ be the rectangle $A=$ $[0,1] \times[-1,1]$. For $(\alpha, \beta) \in A$ put

$$
x_{\alpha, \beta}=\alpha\left(I-Q_{j}\right) x+\beta Q_{j} n_{j}
$$

Let $G=\left\{(\alpha, \beta) \in A: x_{\alpha, \beta} \in B\right\}$ be the set of 'good' indices. The set $G$ is obviously non-empty (since $(0,0) \in G$ ) and closed (since $B$ is closed). The proof will be complete if we check that $G=A$.

With the convex closed set $B$ we can associate the projection pair $\left(P_{B}, Q_{B}\right)$ defined as follows. For a given $x \in X$, define $w=Q_{B} x$ and $y=P_{B} x=x-Q_{B} x$ by the formula

$$
w \in B, \quad|y|=\min \{|x-z|: z \in B\} .
$$

As a consequence of the definition, the point $y=P_{B} x$ belongs to the outward normal cone $\mathcal{N}_{B}(w)$. Let $(\bar{\alpha}, \bar{\beta}) \in G$ be given such that $0 \leq \bar{\alpha}<1$ and $-1<\bar{\beta}<1$. We choose arbitrary $(\alpha, \beta) \in A$ such that

$$
|\beta|+|\alpha-\bar{\alpha}|\left|\left(I-Q_{j}\right) x\right|+\frac{|\beta-\bar{\beta}|}{\left\langle r_{j}, n_{j}\right\rangle}<1
$$

and put $w_{\alpha, \beta}=Q_{B} x_{\alpha, \beta}$ and $y_{\alpha, \beta}=P_{B} x_{\alpha, \beta}$. Then

$$
\begin{aligned}
\left|\left\langle w_{\alpha, \beta}, n_{j}\right\rangle\right| & \leq\left|\left\langle x_{\alpha, \beta}, n_{j}\right\rangle\right|+\left|\left\langle y_{\alpha, \beta}, n_{j}\right\rangle\right| \\
& \leq|\beta|+\left|y_{\alpha, \beta}\right| \\
& \leq|\beta|+\left|x_{\alpha, \beta}-x_{\bar{\alpha}, \bar{\beta}}\right| \\
& \leq|\beta|+|\alpha-\bar{\alpha}|\left|\left(I-Q_{j}\right) x\right|+\frac{|\beta-\bar{\beta}|}{\left\langle r_{j}, n_{j}\right\rangle} .
\end{aligned}
$$

From (4.2) $\left|\left\langle w_{\alpha, \beta}, n_{j}\right\rangle\right|<1$ follows, and Condition $(\mathcal{B})$ yields

$$
\left\langle y_{\alpha, \beta}, r_{j}\right\rangle=0 \text {. }
$$

On the other hand, by definition of the outward normal cone, $\left\langle y_{\alpha, \beta}, w_{\alpha, \beta}-w\right\rangle \geq 0$ for all $w \in B$. We can choose in particular $w=\alpha x$, and from (4.3) we obtain

$$
0 \leq\left\langle y_{\alpha, \beta}, w_{\alpha, \beta}-\alpha x\right\rangle=\left\langle y_{\alpha, \beta}, w_{\alpha, \beta}-x_{\alpha, \beta}\right\rangle=-\left|y_{\alpha, \beta}\right|^{2} .
$$

We conclude that $x_{\alpha, \beta} \in B$, hence the set $G$ is relatively open in $A$. Therefore $G=A$, and Theorem 4.1 is proved

We now give some useful consequences of Theorem 4.1.

Corollary 4.3. Let Hypothesis 1.2 hold and let $B$ be $\mathcal{Q}$-invariant. Then

$$
\left\langle z, n_{j}\right\rangle\left\langle y, r_{j}\right\rangle \geq 0
$$

for all $z \in B, y \in \mathcal{N}_{B}(z)$ and $j \in J$.

Proof. Let $j \in J, z \in B$ and $y \in \mathcal{N}_{B}(z)$ be given. We have $\langle y, z-w\rangle \geq 0$ for every $w \in B$. Using Theorem 4.1 we obtain the assertion by putting $w=\left(I-Q_{j}\right) z$ 
The following result is immediate and we leave the proof to the reader.

Corollary 4.4. Let $B$ be a $\mathcal{Q}$-invariant set. Then the sets $\varrho B=\{\varrho x: x \in B\}$ are $\mathcal{Q}$-invariant for every $\varrho \in \mathbb{R}$ with $|\varrho| \geq 1$. Moreover, if $B_{1}$ and $B_{2}$ are $\mathcal{Q}$-invariant, then $B^{*}=\operatorname{conv}\left(B_{1} \cup B_{2}\right)$ and $B_{*}=B_{1} \cap B_{2}$ are $\mathcal{Q}$-invariant. In particular, to every $\mathcal{Q}$-invariant set $B$ there exists a symmetric $\mathcal{Q}$-invariant set $B_{\mathrm{sym}}=B \cap(-B)$.

We now give an explicit description of the minimal $\mathcal{Q}$-invariant set. The construction is illustrated on Figure 2 in Section 8.

Corollary 4.5. Let $\Lambda$ denote the set of all finite sequences $\lambda=\left(j_{0}, \ldots, j_{m-1}\right)(m \in$ $\mathbb{N})$ such that $j_{k} \in J$ for $k=0, \ldots, m-1$. Let $s_{\lambda}=\left(x_{0}, \ldots, x_{m}\right)$ be the sequence

$$
\left.\begin{array}{rl}
x_{0} & =0 \\
x_{k+1} & =\left(I-Q_{j_{k}}\right) x_{k} \pm Q_{j_{k}} n_{j_{k}} \quad(k=0, \ldots, m-1)
\end{array}\right\}
$$

and put $x_{\lambda}^{\omega}=x_{m}$. Further, let $B^{\omega}$ be the set $B^{\omega}=\overline{\operatorname{conv}}\left\{x_{\lambda}^{\omega}: \lambda \in \Lambda\right\}$. Then:

(i) $B^{\omega}$ is a symmetric $\mathcal{Q}$-invariant set.

(ii) Every $\mathcal{Q}$-invariant set $B$ contains $B^{\omega}$.

Proof. To prove assertion (i) it suffices to check that $B^{\omega}$ satisfies $(\mathcal{A})$. By definition of $B^{\omega},\left(I-Q_{j}\right) x_{\lambda}^{\omega} \pm Q_{j} n_{j} \in B^{\omega}$ for every $j \in J$ and every $\lambda \in \Lambda$. In a similar way, for every convex combination

$$
x=\sum_{i=1}^{n} \alpha_{i} x_{\lambda_{i}}^{\omega} \in B^{\omega} \quad \text { with } \sum_{i=1}^{n} \alpha_{i}=1 \text { and } \alpha_{i} \geq 0
$$

we have

$$
\left(I-Q_{j}\right) x \pm Q_{j} n_{j}=\sum_{i=1}^{n} \alpha_{i}\left(\left(I-Q_{j}\right) x_{\lambda_{i}}^{\omega} \pm Q_{j} n_{j}\right) \in B^{\omega}
$$

and the closedness of $B^{\omega}$ yields assertion (i).

Assertion (ii) is an immediate consequence of Theorem 4.1: if $B$ is a $\mathcal{Q}$-invariant set, then by induction $x_{\lambda}^{\omega} \in B$ for every $\lambda \in \Lambda$. Since $B$ is convex and closed, the assertion follows

Remark 4.6. A sequence $s_{\lambda}$ of form (4.4) is called an 1-trajectory associated to $\lambda \in \Lambda$. We will see below in Theorem 5.8 that the Lipschitz constant of the Skorokhod map is related to the diameter of the set $B$ from Theorem 4.1. According to Corollary $4.5, B^{\omega}$ is the minimal set with the desired property. An upper bound for all possible 1-trajectories will therefore yield an upper bound for the Lipschitz constant.

In particular, we have to ask whether $B^{\omega}$ is bounded. We first state a necessary condition in terms of the vectors $n_{j}$ and $r_{j}$. For each $J^{\prime} \subset J$ we define the spaces

$$
\left.\begin{array}{l}
R_{J^{\prime}}=\operatorname{span}\left\{r_{j}: j \in J^{\prime}\right\} \\
N_{J^{\prime}}=\operatorname{span}\left\{n_{j}: j \in J^{\prime}\right\}
\end{array}\right\}
$$


Lemma 4.7. For every $J^{\prime} \subset J$ we have

$$
R_{J^{\prime}} \cap N_{J^{\prime}}^{\perp} \subset B^{\omega} \subset R_{J}
$$

where $R_{J^{\prime}}$ and $N_{J^{\prime}}$ are defined by (4.5) and $N_{J^{\prime}}^{\perp}$ denotes the orthogonal complement to $N_{J^{\prime}}$.

Proof. The fact that $B^{\omega} \subset R_{J}$ is obvious. Let $J^{\prime} \subset J$ and $x \in R_{J^{\prime}} \cap N_{J^{\prime}}^{\perp}$ be arbitrarily chosen and assume $x \neq 0$. We find real numbers $a_{i}\left(i \in J^{\prime}\right)$ such that $x=\sum_{i \in J^{\prime}} a_{i} r_{i}$ and put $b_{i}=\left\langle n_{i}, r_{i}\right\rangle a_{i}$ and $c=\sum_{i \in J^{\prime}}\left|b_{i}\right|$. Then the point

$$
\frac{1}{c} x=\sum_{i \in J^{\prime}} \frac{1}{c} b_{i} Q_{i} n_{i}
$$

belongs to $B^{\omega}$ by definition. Moreover, if $k x \in B^{\omega}$ for some $k \in \mathbb{R}$, then by Theorem 4.1 and Corollary 4.5

$$
\left(I-Q_{j}\right) k x \pm \operatorname{sign}\left(b_{j}\right) Q_{j} n_{j} \in B^{\omega} \quad \forall j \in J^{\prime}
$$

By hypothesis, $Q_{j} x=0$ for every $j \in J^{\prime}$, and the convexity of $B^{\omega}$ yields

$$
\begin{aligned}
\sum_{j \in J^{\prime}} \frac{1}{c}\left|b_{j}\right|\left(\left(I-Q_{j}\right) k x \pm \operatorname{sign}\left(b_{j}\right) Q_{j} n_{j}\right) & =\sum_{j \in J^{\prime}} \frac{1}{c}\left|b_{j}\right|\left(k x \pm \operatorname{sign}\left(b_{j}\right) Q_{j} n_{j}\right) \\
& =\left(k \pm \frac{1}{c}\right) x \in B^{\omega}
\end{aligned}
$$

hence $B^{\omega}$ contains the whole line $\operatorname{span}\{x\}$

Corollary 4.8. Let $B^{\omega}$ be bounded. Then

$$
R_{J^{\prime}} \cap N_{J^{\prime}}^{\perp}=\{0\}
$$

for all $J^{\prime} \subset J$.

In the sequel, condition (4.6) will be referred to as the transversality condition. It is obviously satisfied in the case of normal reflection and, obviously as well, it is not robust with respect to small changes of reflection vectors. This is indeed a drawback, but we show below in Corollary 5.3 that in combination with $\ell$-paracontractivity the transversality condition is equivalent to the condition

$$
\operatorname{dim} N_{J^{\prime}}=\operatorname{dim} R_{J^{\prime}} \quad \forall J^{\prime} \subset J
$$

which is simply a linear constraint to the robustness of the $\ell$-paracontractivity.

For the reader's convenience, we give here the proof of the following Lipschitz estimate which basically follows the lines of [3: Theorem 2.2]. We however do not assume explicitly here that the set $B$ has non-empty interior. 
Theorem 4.9. Let Hypothesis 1.2 hold and let there exist a symmetric $\mathcal{Q}$-invariant set $B$. Let $m_{B}: X \rightarrow \mathbb{R}^{+} \cup\{+\infty\}$ be the Minkowski functional of the set $B$, that is,

$$
m_{B}(x)=\inf \left\{s>0: \frac{1}{s} x \in B\right\} \quad(x \in X) .
$$

Let $u_{1}, u_{2} \in C([0, T] ; X)$ be two input functions for which there exist respective solutions $\left(\xi_{1}, x_{1}\right),\left(\xi_{2}, x_{2}\right)$ to the Skorokhod problem. For $t \in[0, T]$ put

$$
\begin{aligned}
& \bar{\xi}(t)=\xi_{1}(t)-\xi_{2}(t) \\
& \bar{x}(t)=x_{1}(t)-x_{2}(t) \\
& \bar{u}(t)=u_{1}(t)-u_{2}(t) .
\end{aligned}
$$

Then for every $t \in[0, T]$ we have

$$
m_{B}(\bar{\xi}(t)) \leq \max \left\{m_{B}(\bar{\xi}(0)),|\bar{u}|_{[0, t]}\right\} .
$$

Proof. Put $X_{B}=\left\{x \in X: m_{B}(x)<\infty\right\}$. Then $X_{B}$ is a subspace of $X$, and since $\pm Q_{j} n_{j} \in B^{\omega}$ for every $j \in J$, we obtain from Corollary 4.5 that $R_{J} \subset X_{B}$.

The statement is empty if $\bar{\xi}(0) \notin X_{B}$. Let us assume therefore that $\bar{\xi}(0) \in X_{B}$, and for $t \in[0, T]$ put $\gamma(t)=|\bar{u}|_{[0, t]}$. For every $t \in[0, T]$ we have by definition

$$
\bar{\xi}(t)-\bar{\xi}(0) \in \mathcal{R}_{[0, t]}\left(x_{1}\right)-\mathcal{R}_{[0, t]}\left(x_{2}\right) \subset X_{B},
$$

hence we can restrict our considerations to the reduced Minkowski functional

$$
\tilde{m}_{B}=\left.m_{B}\right|_{X_{B}} .
$$

For $t \in[0, T]$ put $\psi(t)=\tilde{m}_{B}(\bar{\xi}(t))$ and assume the assertion of Theorem 4.9 does not hold. We can find $t_{0} \in(0, T)$ such that $\gamma_{0}:=\psi\left(t_{0}\right)>\gamma\left(t_{0}\right)$ and $\psi(t)<\psi\left(t_{0}\right)$ for $t \in\left[0, t_{0}\right)$. Put $z=\bar{\xi}\left(t_{0}\right) / \gamma_{0}$. Then $z \in B$ and, for every $y \in \partial \tilde{m}_{B}(z)$ where $\partial \tilde{m}_{B}$ is the subdifferential of $\tilde{m}_{B}$, by definition

$$
\langle y, z-\tilde{z}\rangle \geq \tilde{m}_{B}(z)-\tilde{m}_{B}(\tilde{z}) \quad \forall \tilde{z} \in X_{B} .
$$

In particular, $y \in \mathcal{N}_{B}(z)$, and putting $\tilde{z}=\bar{\xi}\left(t_{0}-h\right) / \gamma_{0}$ in (4.9) for small positive $h$ we obtain

$$
\left\langle y, \bar{\xi}\left(t_{0}\right)-\bar{\xi}\left(t_{0}-h\right)\right\rangle \geq \gamma_{0}\left(\psi\left(t_{0}\right)-\psi\left(t_{0}-h\right)\right)>0 .
$$

By (1.3) choose $h$ sufficiently small such that

$$
\left.\begin{array}{l}
\tilde{J}\left(x_{1}(t)\right) \subset \tilde{J}\left(x_{1}\left(t_{0}\right)\right) \\
\tilde{J}\left(x_{2}(t)\right) \subset \tilde{J}\left(x_{2}\left(t_{0}\right)\right)
\end{array}\right\} \quad\left(t \in\left[t_{0}-h, t_{0}\right]\right) .
$$

By (1.7), we have

$$
\begin{aligned}
& \xi_{1}\left(t_{0}\right)-\xi_{1}\left(t_{0}-h\right) \in \mathcal{C}\left(\tilde{J}\left(x_{1}\left(t_{0}\right)\right)\right) \\
& \xi_{2}\left(t_{0}\right)-\xi_{2}\left(t_{0}-h\right) \in \mathcal{C}\left(\tilde{J}\left(x_{2}\left(t_{0}\right)\right)\right) .
\end{aligned}
$$

We thus infer from (4.10) that there exists either some $j \in \tilde{J}\left(x_{1}\left(t_{0}\right)\right)$ such that $\left\langle y, r_{j}\right\rangle>$ 0 , or some $i \in \tilde{J}\left(x_{2}\left(t_{0}\right)\right)$ such that $\left\langle y, r_{i}\right\rangle<0$. Both cases are symmetric, let us assume therefore $\left\langle y, r_{j}\right\rangle>0$ for some $j \in \tilde{J}\left(x_{1}\left(t_{0}\right)\right)$. Then Corollary 4.3 yields $\left\langle z, n_{j}\right\rangle \geq 0$. On the other hand, by definition of $\tilde{J}\left(x_{1}\left(t_{0}\right)\right)$ we have $\left\langle\bar{x}\left(t_{0}\right), n_{j}\right\rangle \geq 0$. We conclude with

$$
0 \leq\left\langle z, n_{j}\right\rangle=\frac{1}{\gamma_{0}}\left\langle\bar{u}\left(t_{0}\right), n_{j}\right\rangle-\frac{1}{\gamma_{0}}\left\langle\bar{x}\left(t_{0}\right), n_{j}\right\rangle \leq \frac{1}{\gamma_{0}}\left\langle\bar{u}\left(t_{0}\right), n_{j}\right\rangle \leq \frac{\gamma\left(t_{0}\right)}{\gamma_{0}}<1 .
$$

This violates property $(\mathcal{B})$ from Theorem 4.1 , which is indeed a contradiction. Theorem 4.9 is proved 
For practical purposes, formula (4.8) is more convenient to work with if the set $B$ has non-empty interior. The following straightforward argument shows that this condition represents no restriction.

Proposition 4.10. Let $B$ be a $\mathcal{Q}$-invariant set and let $B_{1}(0)$ denote the unit ball in $X$. Then $B^{\prime}=2 B+B_{1}(0)$ is also a $\mathcal{Q}$-invariant set.

Proof. Let $x^{\prime} \in B^{\prime}$ and $y \in \mathcal{N}_{B^{\prime}}\left(x^{\prime}\right)$ be given such that $\left|\left\langle x^{\prime}, n_{j}\right\rangle\right|<1$ for some $j \in J$. There exist $x \in B$ and $h \in B_{1}(0)$ such that $x^{\prime}=2 x+h$. By definition of the normal cone, $\left\langle y, x^{\prime}-(2 b+h)\right\rangle \geq 0$ for every $b \in B$, hence $y \in \mathcal{N}_{B}(x)$. On the other hand, $\left|\left\langle x, n_{j}\right\rangle\right|=\frac{1}{2}\left|\left\langle x^{\prime}-h, n_{j}\right\rangle\right|<1$. Since $B$ is $\mathcal{Q}$-invariant we obtain $\left\langle y, r_{j}\right\rangle=0$ and the proof is complete

Corollary 4.11. If there exists a bounded $\mathcal{Q}$-invariant set, then there exists a bounded $\mathcal{Q}$-invariant set with non-empty interior.

Theorem 4.9 implies uniqueness of solutions and a Lipschitz continuous dependence with respect to the sup-norm provided the set $B$ is bounded. Existence (in $\left.W^{1,1}(0, T ; X)\right)$ and uniqueness (in $\left.C([0, T] ; X)\right)$ thus have been proved under different hypotheses. In the next Section 5 we show (Theorem 5.5 ) that the $\ell$-paracontractivity together with transversality of the system $\mathcal{Q}$ ensures the existence of a bounded $\mathcal{Q}$ invariant set. This will enable us to characterize a class of Skorokhod problems for which existence, uniqueness and Lipschitz continuous dependence hold.

\section{Paracontractivity and invariant sets}

Keeping the notation from Corollary 4.5, we assume that $\mathcal{Q}$ is an $\ell$-paracontracting system, and that $x \in X$ and $\lambda \in \Lambda, \lambda=\left(j_{0}, \ldots, j_{m-1}\right)$ are given. Let us consider the sequence

$$
\left.\begin{array}{rl}
x_{0} & =x \\
x_{k+1} & =\left(I-Q_{j_{k}}\right) x_{k} \quad(k=0,1, \ldots, m-1)
\end{array}\right\} .
$$

We define the mapping $\omega_{\lambda}: X \rightarrow X$ by

$$
\omega_{\lambda}(x)=x_{m}
$$

By definition of $\ell$-paracontractivity,

$$
\left|x_{k+1}-x_{k}\right| \leq\left\|x_{k}\right\|-\left\|x_{k+1}\right\| \quad(k=0,1, \ldots, m-1),
$$

hence

$$
\left|x-\omega_{\lambda}(x)\right| \leq\|x\|-\left\|\omega_{\lambda}(x)\right\| .
$$

We now introduce some further notation. For $J^{\prime} \subset J$ put

$$
\Lambda_{J^{\prime}}=\left\{\lambda \in \Lambda: \lambda=\left(j_{0}, \ldots, j_{m-1}\right) \text { with } \bigcup_{k=0}^{m-1}\left\{j_{k}\right\}=J^{\prime}\right\} .
$$

We start with two auxiliary results. 
Lemma 5.1. Let $\mathcal{Q}$ be an $\ell$-paracontracting system, and let $J^{\prime} \subset J$ and $\lambda \in \Lambda_{J^{\prime}}$ be given. Then $\omega_{\lambda}(x)=x$ if and only if $x \in N_{J^{\prime}}^{\perp}$.

Proof. We have indeed $\omega_{\lambda}(x)=x$ for $x \in N_{J^{\prime}}^{\perp}$. Conversely, let $\omega_{\lambda}(x)=x$ for some $x \in X$ and $\lambda \in \Lambda_{J^{\prime}}, \lambda=\left(j_{0}, \ldots, j_{m-1}\right)$. From (5.3) we infer $x=x_{1}=\ldots=x_{m-1}$ and $Q_{j} x=0$ for all $j \in J^{\prime}$, hence $x \in N \frac{\perp}{J^{\prime}}$

Lemma 5.2. Let $\mathcal{Q}$ be an $\ell$-paracontracting system. Then $R_{J^{\prime}}^{\perp} \cap N_{J^{\prime}}=\{0\}$ for every $J^{\prime} \subset J$.

Proof. For arbitrary $z \in R_{J^{\prime}}^{\perp} \cap N_{J^{\prime}}$ and $\lambda \in \Lambda_{J^{\prime}}$ we define recursively the sequence

$$
\left.\begin{array}{l}
z_{0}=z \\
z_{n}=\omega_{\lambda}\left(z_{n-1}\right) \quad(n \in \mathbb{N})
\end{array}\right\} .
$$

By (5.4), $\left|z_{n}-z_{n+1}\right| \leq\left\|z_{n}\right\|-\left\|z_{n+1}\right\|$, hence $\left\{z_{n}\right\}$ is a convergent sequence, $z_{n} \rightarrow z^{*}$. On the other hand, for every $j \in J^{\prime}$ and $x \in X$ we have $\left\langle Q_{j} x, z\right\rangle=0$, hence $\left\langle z_{n}, z\right\rangle=|z|^{2}$ for every $n \in \mathbb{N}$. Passing to the limit as $n \rightarrow \infty$ we obtain $z^{*}=\omega_{\lambda}\left(z^{*}\right)$ and $\left\langle z^{*}, z\right\rangle=|z|^{2}$, hence by Lemma $5.1 z^{*} \in N_{J^{\prime}}^{\perp}$ and $z=0$

As an immediate consequence of Lemma 5.2 we get

Corollary 5.3. Let $\mathcal{Q}$ be an $\ell$-paracontracting system. Then the following conditions are equivalent:

(i) Transversality condition (4.6) holds.

(ii) Condition (4.7) holds.

(iii) $R_{J^{\prime}}^{\perp} \oplus N_{J^{\prime}}=R_{J^{\prime}} \oplus N_{J^{\prime}}^{\perp}=X$ for every $J^{\prime} \subset J$.

The next statement is the key point of this section and illustrates the meaning of paracontractivity. We see that for every $J^{\prime} \subset J$ and $\lambda \in \Lambda_{J^{\prime}}$ the mapping $\omega_{\lambda}$ leaves invariant both complementary subspaces $R_{J^{\prime}}$ and $N_{J^{\prime}}^{\perp}$, and that it reduces to the identity on $N_{J^{\prime}}^{\perp}$ and to a contraction on $R_{J^{\prime}}$ with respect to the norm $\|\cdot\|$.

Proposition 5.4. Let $\mathcal{Q}$ be an $\ell$-paracontracting system and let transversality condition (4.6) hold. Then for every $J^{\prime} \subset J$ there exists $\delta_{J^{\prime}} \in[0,1)$ such that

$$
\omega_{\lambda}(x) \in R_{J^{\prime}}, \quad\left\|\omega_{\lambda}(x)\right\| \leq \delta_{J^{\prime}}\|x\| .
$$

for all $x \in R_{J^{\prime}}$ and all $\lambda \in \Lambda_{J^{\prime}}$.

Proof. Let $J^{\prime} \subset J$ be given. The fact that $\omega_{\lambda}(x) \in R_{J^{\prime}}$ for $x \in R_{J^{\prime}}$ and $\lambda \in \Lambda_{J^{\prime}}$ is obvious. Put

$$
\delta_{J^{\prime}}=\sup \left\{\left\|\omega_{\lambda}(x)\right\|: \lambda \in \Lambda_{J^{\prime}} \text { and } x \in R_{J^{\prime}} \text { with }\|x\|=1\right\} .
$$

By (5.4) we have $\delta_{J^{\prime}} \leq 1$. Assume $\delta_{J^{\prime}}=1$. Then there exists a sequence $\left\{x_{n}\right\}_{n \in \mathbb{N}}$ in $R_{J^{\prime}}$ with $\left\|x_{n}\right\|=1$ and a sequence $\left\{\lambda_{n}\right\}_{n \in \mathbb{N}}$ in $\Lambda_{J^{\prime}}$ such that

$$
\left\|\omega_{\lambda_{n}}\left(x_{n}\right)\right\| \geq 1-\frac{1}{n} \quad(n \in \mathbb{N}) .
$$


We may assume $x_{n} \rightarrow x$ with $\|x\|=1$. Let us fix an arbitrary $j \in J^{\prime}$. For each $n \in \mathbb{N}$, the sequence $\lambda_{n}=\left(j_{0}^{(n)}, \ldots, j_{m_{n}-1}^{(n)}\right)$ contains $j$, say $j=j_{k_{n}}^{(n)}$ for some $k_{n} \leq m_{n}-1$. Put $\lambda_{n}^{\prime}=\left(j_{0}^{(n)}, \ldots, j_{k_{n}-1}^{(n)}\right)$ and $z_{n}=\omega_{\lambda_{n}^{\prime}}\left(x_{n}\right)$. Then, by $(5.3)$,

$$
\begin{aligned}
\left\|\omega_{\lambda_{n}}\left(x_{n}\right)\right\| & \leq\left\|\left(I-Q_{j}\right) z_{n}\right\| \\
\left\|z_{n}\right\| & \leq\left\|x_{n}\right\|=1
\end{aligned}
$$

for every $n \in \mathbb{N}$, hence

$$
\left\|z_{n}\right\|-\left|Q_{j} z_{n}\right| \geq\left\|\left(I-Q_{j}\right) z_{n}\right\| \geq 1-\frac{1}{n} \quad(n \in \mathbb{N})
$$

Therefore $\lim _{n \rightarrow \infty}\left\|z_{n}\right\|=1$ and $\lim _{n \rightarrow \infty}\left|Q_{j} z_{n}\right|=0$, and (5.3) entails

$$
\left|x_{n}-z_{n}\right| \leq\left\|x_{n}\right\|-\left\|z_{n}\right\| \quad(n \in \mathbb{N})
$$

We conclude that $\lim _{n \rightarrow \infty} z_{n}=x$ and $Q_{j} x=0$ for all $j \in J^{\prime}$, which contradicts transversality condition (4.6)

The main result of this section can be stated as follows.

Theorem 5.5. Let $\mathcal{Q}$ be an $\ell$-paracontracting system and let transversality condition (4.6) hold. Then the minimal $\mathcal{Q}$-invariant set $B^{\omega}$ from Corollary 4.5 is contained in the ball centered at 0 of radius $K$ with respect to the norm $\|\cdot\|$, where

$$
K \leq \frac{C}{\delta}\left(\left(\frac{1}{1-\delta}\right)^{p}-1\right)
$$

with $C=\max \left\{\frac{\left\|r_{j}\right\|}{\left\langle n_{j}, r_{j}\right\rangle}: j \in J\right\}$ and any $\delta \in(0,1)$ with $\delta \geq \max \left\{\delta_{J^{\prime}}: J^{\prime} \subset J\right\}$.

We postpone the proof of Theorem 5.5 and prove first an auxiliary statement.

Proposition 5.6. Let the assumptions of Theorem 5.5 hold, and let $J^{\prime} \subset J$ and $\lambda \in \Lambda_{J^{\prime}}$ be given, card $J^{\prime}=q \in J$ and $\lambda=\left(j_{0}, \ldots, j_{m-1}\right)$. Let $\hat{n}_{j_{k}}= \pm n_{j_{k}}$ be arbitrarily chosen for each $k=0, \ldots, m-1$ and let us define the sequence

$$
\left.\begin{array}{rl}
z_{k} & =\left(I-Q_{j_{m-1}}\right) \cdots\left(I-Q_{j_{k}}\right) Q_{j_{k-1}} \hat{n}_{j_{k-1}} \quad(k=1, \ldots, m-1) \\
z_{m} & =Q_{j_{m-1}} \hat{n}_{j_{m-1}}
\end{array}\right\} .
$$

Then

$$
\left\|\sum_{k=1}^{m} z_{k}\right\| \leq \frac{C}{\delta}\left(\left(\frac{1}{1-\delta}\right)^{q}-1\right) .
$$

The proof of Proposition 5.6 is based on the following induction step. 
Lemma 5.7. Let the assertion of Proposition 5.6 hold for some $q<p$, and let $J^{\prime} \subset J$ and $\lambda \in \Lambda_{J^{\prime}}$ be given, card $J^{\prime}=q+1$ and $\lambda=\left(j_{0}, \ldots, j_{m-1}\right)$, such that $\lambda^{\prime}=\left(j_{1}, \ldots, j_{m-1}\right) \notin \Lambda_{J^{\prime}}$. Let $z_{k}$ be defined by $(5.12)$. Then

$$
\left\|\sum_{k=1}^{m} z_{k}\right\| \leq C\left(1+\frac{1}{\delta}\left(\left(\frac{1}{1-\delta}\right)^{q}-1\right)\right) .
$$

Proof. By induction hypothesis, we have

$$
\left\|\sum_{k=2}^{m} z_{k}\right\| \leq \frac{C}{\delta}\left(\left(\frac{1}{1-\delta}\right)^{q}-1\right)
$$

while

$$
\left\|z_{1}\right\| \leq\left\|Q_{j_{0}} \hat{n}_{j_{0}}\right\| \leq C,
$$

and formula (5.14) follows easily

Proof of Proposition 5.6. For $q=1$ we have $z_{k}=0$ for $k<m$, hence

$$
\left\|\sum_{k=1}^{m} z_{k}\right\|=\left\|z_{m}\right\| \leq C
$$

and (5.13) holds. Assume now that the assertion holds for some $q \geq 1, q<p$ and fix some $J^{\prime} \subset J$, card $J^{\prime}=q+1$, and $\lambda \in \Lambda_{J^{\prime}}, \lambda=\left(j_{0}, \ldots, j_{m-1}\right)$. We define the numbers $d(0), d(1), \ldots, d(\ell)$ recurrently according to the following recipe:

$$
\left.\begin{array}{rl}
d(0) & =m \\
d(1) & =\max \left\{k<m:\left(j_{k}, \ldots, j_{m-1}\right) \in \Lambda_{J^{\prime}}\right\} \\
& \vdots \\
d(n+1) & =\max \left\{k<d(n):\left(j_{k}, \ldots, j_{d(n)-1}\right) \in \Lambda_{J^{\prime}}\right\}
\end{array}\right\}
$$

until $\left(j_{0}, \ldots, j_{d(\ell)-1}\right) \notin \Lambda_{J^{\prime}}$. For $n=0, \ldots, \ell$ and $k=1, \ldots, d(n)-1$ put

$$
\left.\begin{array}{rl}
\zeta_{k}^{n} & =\left(I-Q_{j_{d(n)-1}}\right) \cdots\left(I-Q_{j_{k}}\right) Q_{j_{k-1}} \hat{n}_{j_{k-1}} \\
\zeta_{d(n)}^{n} & =Q_{j_{d(n)-1}} \hat{n}_{j_{d(n)-1}}
\end{array}\right\} .
$$

Then for $d(n+1)+1 \leq k \leq d(n)$ we have

$$
z_{k}=\left(I-Q_{j_{m-1}}\right) \cdots\left(I-Q_{j_{d(n)}}\right) \zeta_{k}^{n}
$$

The inequality

$$
\left\|\sum_{k=d(n+1)+1}^{d(n)} \zeta_{k}^{n}\right\| \leq C\left(1+\frac{1}{\delta}\left(\left(\frac{1}{1-\delta}\right)^{q}-1\right)\right)
$$


where we put $d(\ell+1)=0$, is valid for $n=0, \ldots, \ell-1$ according to Lemma 5.7 and for $n=\ell$ according to the induction hypothesis. Proposition 5.4 now yields for $n=0, \ldots, \ell$

$$
\begin{aligned}
\left\|\sum_{k=d(n+1)+1}^{d(n)} z_{k}\right\| & =\left\|\left(I-Q_{j_{m-1}}\right) \cdots\left(I-Q_{j_{d(n)}}\right) \sum_{k=d(n+1)+1}^{d(n)} \zeta_{k}^{n}\right\| \\
& \leq C \delta^{n}\left(1+\frac{1}{\delta}\left(\left(\frac{1}{1-\delta}\right)^{q}-1\right)\right) .
\end{aligned}
$$

Summing up the above inequalities over $n=0, \ldots, \ell$ we obtain

$$
\left\|\sum_{k=1}^{m} z_{k}\right\| \leq \frac{C}{1-\delta}\left(1+\frac{1}{\delta}\left(\left(\frac{1}{1-\delta}\right)^{q}-1\right)\right)=\frac{C}{\delta}\left(\left(\frac{1}{1-\delta}\right)^{q+1}-1\right)
$$

and the induction step is complete. Proposition 5.6 is proved

We are now ready to conclude this section by proving Theorem 5.5.

Proof of Theorem 5.5. Let $\lambda \in \Lambda, \lambda=\left(j_{0}, \ldots, j_{m-1}\right)$ be arbitrary, and let $s_{\lambda}$ be the corresponding 1-trajectory defined by (4.4). Then

$$
\left.\begin{array}{l}
x_{1}=Q_{j_{0}} \hat{n}_{j_{0}} \\
x_{k}=\sum_{i=1}^{k-1}\left(I-Q_{j_{k-1}}\right) \cdots\left(I-Q_{j_{i}}\right) Q_{j_{i-1}} \hat{n}_{j_{i-1}} \quad(k=2, \ldots, m-1)
\end{array}\right\}
$$

for some $\hat{n}_{j_{i}}= \pm n_{j_{i}} \quad(i=0, \ldots, m-1)$. Using Proposition 5.6 we obtain

$$
\sup _{\lambda \in \Lambda}\left\|x_{\lambda}^{\omega}\right\| \leq \frac{C}{\delta}\left(\left(\frac{1}{1-\delta}\right)^{p}-1\right)
$$

hence inequality (5.11) holds

Theorem 5.8. Let the associated projection system $\mathcal{Q}$ be $\ell$-paracontracting and transversal. Then the Skorokhod map $\mathcal{S}$ is well defined and of Lipschitz type both as a map from $Z \times W^{1,1}(0, T ; X)$ to $W^{1,1}(0, T ; X)$ and from $Z \times C([0, T] ; X)$ to $C([0, T] ; X)$.

Proof. Theorem 3.1 guarantees that the Skorokhod problem admits a solution for every $u \in W^{1,1}(0, T ; X)$ and every initial condition. By Theorem 5.5 , the set $B^{\omega}$ is bounded. There exists therefore $M>0$ such that $B^{\omega}$ is contained in a ball centered at 0 with radius $M$. Using the fact that the space $W^{1,1}(0, T ; X)$ is dense in $C([0, T] ; X)$, we obtain the existence and Lipschitz continuity in $C([0, T] ; X)$ immediately from Theorem 4.9 , from the upper semicontinuity property (1.6) and from the inequality $m_{B^{\omega}}(x) \geq \frac{|x|}{M}$ for every $x \in X$. The Lipschitz continuity in $Z \times W^{1,1}(0, T ; X) \rightarrow W^{1,1}(0, T ; X)$ follows immediately from Remark 3.2 


\section{A bounded variation result}

Similarly as in the normal reflection case, one might expect that, if the set $B$ in Theorem 4.9 is bounded and $Z$ has non-empty interior, the extension of the Skorokhod map onto $C([0, T] ; X)$ has a regularizing effect, namely that for inputs $u \in C([0, T] ; X)$ the outputs $\xi$ belong to $C([0, T] ; X) \cap B V(0, T ; X)$.

Assume that there exists $z_{0} \in Z$ and $\varrho>0$ such that the whole ball $B_{\varrho}\left(z_{0}\right)$ is contained in $Z$. We prove the following result (which subsequently immediately implies the desired $B V$-estimate).

Proposition 6.1. Let the associated projection system $\mathcal{Q}$ be -paracontracting and transversal. Let $u \in C([0, T] ; X)$ be given and let $\xi, x \in C([0, T] ; X)$ be the corresponding solution to the Skorokhod problem for a given initial condition $x_{0} \in Z$. Let $\delta>0$ be such that the implication

$$
\left|t_{2}-t_{1}\right|<\delta \quad \Longrightarrow \quad\left|u\left(t_{2}\right)-u\left(t_{1}\right)\right|<\frac{\varrho}{2}
$$

holds for every $t_{1}, t_{2} \in[0, T]$. Then for every $0 \leq s<t \leq T$ such that $|t-s|<\delta$ we have

$$
\operatorname{Var}_{[s, t]} \xi \leq\left\|x(\cdot)-z_{0}\right\|_{[0, t]}
$$

where $\|\cdot\|_{[0, t]}$ denotes the sup-norm with respect to the norm $\|\cdot\|$ over the interval $[0, t]$.

Proof. We approximate the function $u$ uniformly by functions from $W^{1,1}(0, T ; X)$, and for each of these approximating functions we apply the discretization procedure from Section 3. By diagonalization we obtain, according to Theorem 5.8 and to the construction in the proof of Theorem 3.1, discrete sequences $\left\{u_{i}\right\},\left\{x_{i}\right\},\left\{\xi_{i}\right\}$ satisfying (3.1) such such that the piecewise linear interpolates $\left\{u^{(n)}\right\},\left\{x^{(n)}\right\},\left\{\xi^{(n)}\right\}$ given by (3.6) converge uniformly to $u, x, \xi$, respectively.

Let $\varepsilon>0$ be arbitrarily given. We find $n_{0}$ sufficiently large such that for $n>n_{0}$ we have $\left|u^{(n)}-u\right|_{[0, T]}<\frac{\varrho}{4}$ and $\left\|x^{(n)}-x\right\|_{[0, T]}<\varepsilon$, and there exist $t_{j-1}^{(n)} \leq s<t \leq t_{k}^{(n)}$ such that $t_{k}^{(n)}-t_{j-1}^{(n)}<\delta$. For $i=j, \ldots, k$ we have by hypothesis

$$
\left|u_{i}-u_{j-1}\right| \leq 2\left|u^{(n)}-u\right|_{[0, T]}+\frac{\varrho}{2} \leq \varrho
$$

hence $z_{i}=z_{0}+u_{i}-u_{j-1} \in Z$ for every $i=j, \ldots, k$. Inequality (3.3) for $z=z_{i}$ yields

$$
\left|\xi_{i}-\xi_{i-1}\right| \leq\left\|x_{i-1}-u_{i-1}+u_{j-1}-z_{0}\right\|-\left\|x_{i}-u_{i}+u_{j-1}-z_{0}\right\|
$$

for all $i=j, \ldots, k$. Summing up the above inequalities we obtain

$$
\operatorname{Var}_{[s, t]} \xi^{(n)} \leq \sum_{i=j}^{k}\left|\xi_{i}-\xi_{i-1}\right| \leq\left\|x_{j}-z_{0}\right\| \leq \varepsilon+\left\|x(\cdot)-z_{0}\right\|_{[0, t]}
$$

Passing to the limit as $n \rightarrow \infty$ and using the fact that $\varepsilon$ has been chosen arbitrarily, we complete the proof 


\section{An upper bound for the invariant sets}

According to Lemma 2.2, the $\ell$-paracontracting property is robust with respect to small changes of vectors $r_{i}$ if the vectors $n_{i}$ do not change. This allows us to extend the Lipschitz continuity results from the normal reflection case to the case of Skorokhod problems with reflection vectors $r_{i}$ that are close to the normals $n_{i}$ under the transversality constraint. This argument, however, does not provide an efficient estimate of the corresponding Lipschitz constant. In this section, we show an algorithm which gives at least an upper bound.

Put $N=\operatorname{dim} N_{J}$ and for $k=1, \ldots, N$ denote

$$
\begin{aligned}
\mathcal{L}_{k} & =\left\{J^{\prime} \subset J: \operatorname{card} J^{\prime}=k \text { and }\left\{n_{i}\right\}_{i \in J^{\prime}} \text { linearly independent }\right\} \\
\varepsilon_{k} & =\min \left\{\left|\sum_{i \in J^{\prime}} \alpha_{i} n_{i}\right|: \sum_{i \in J^{\prime}} \alpha_{i}^{2}=1 \quad\left(J^{\prime} \in \mathcal{L}_{k}\right)\right\} .
\end{aligned}
$$

Note that $0<\varepsilon_{N} \leq \varepsilon_{N-1} \leq \ldots \leq \varepsilon_{1} \leq 1$

We make the following

Hypothesis 7.1. For every $j \in J,\left|n_{j}-r_{j}\right| \leq \frac{\varepsilon_{N}}{2 \sqrt{N}}$ and (4.7) holds. $\frac{7}{8}>0$.

The above hypothesis implies in particular $\left|n_{j}-r_{j}\right|^{2} \leq \frac{1}{4}$ for every $j$, hence $\left\langle n_{j}, r_{j}\right\rangle \geq$

Notation 7.2. For an arbitrary subspace $X^{\prime} \subset X$ we denote by $P_{X^{\prime}}$ the orthogonal projection onto $X^{\prime}$. In particular, $P_{X}=I$ is the identity operator. We further denote by $\mathcal{D}_{k} \quad(0 \leq k \leq N)$ the system of all $k$-dimensional subspaces of $R_{J}$ generated by the vectors $r_{1}, \ldots, r_{p}$, that is

$$
\begin{aligned}
\mathcal{D}_{0}= & \{\{0\}\} \\
\mathcal{D}_{k}= & \left\{X^{\prime} \subset R_{J}: X^{\prime}=\operatorname{span}\left\{r_{i_{1}}, \ldots, r_{i_{m}}\right\} \quad\left(i_{j} \in J \text { for } j=1, \ldots, m\right), \operatorname{dim} X^{\prime}=k\right\} \\
& (k=1, \ldots, N-1) \\
\mathcal{D}_{N}= & \left\{R_{J}\right\} .
\end{aligned}
$$

We need in the sequel the following elementary properties of projections.

Lemma 7.3. Let $X^{\prime} \subset X^{\prime \prime} \subset X$ be subspaces of $X$. Then:

(i) $P_{X^{\prime \prime}} P_{X^{\prime}}=P_{X^{\prime}} P_{X^{\prime \prime}}=P_{X^{\prime}}$.

(ii) $|\langle z, v\rangle| \leq\left|P_{X^{\prime}} z\right| \leq|z|$ for all $z \in X$ and all $v \in X^{\prime}$ with $|v| \leq 1$.

According to Hypothesis 7.1, every system $\left\{r_{i}\right\}_{i \in J^{\prime}}$ for $J^{\prime} \in \mathcal{L}_{k}$ is linearly independent and we may put

$$
\delta_{k}=\min \left\{\left|\sum_{i \in J^{\prime}} \alpha_{i} r_{i}\right|: \sum_{i \in J^{\prime}} \alpha_{i}^{2}=1 \quad\left(J^{\prime} \in \mathcal{L}_{k}\right)\right\}
$$


and have again $0<\delta_{N} \leq \delta_{N-1} \leq \ldots \leq \delta_{1} \leq 1$. Moreover, from Hypothesis 7.1 it follows that

$$
\frac{1}{2} \varepsilon_{k} \leq \delta_{k} \leq \frac{3}{2} \varepsilon_{k} \quad(k=1, \ldots, N)
$$

Indeed, we have for $J^{\prime} \in \mathcal{L}_{k}$

$$
\sum_{i \in J^{\prime}}\left|\alpha_{i}\right|\left|r_{i}-n_{i}\right| \leq \frac{\varepsilon_{N}}{2 \sqrt{N}} \sum_{i \in J^{\prime}}\left|\alpha_{i}\right| \leq \frac{\varepsilon_{N}}{2} \sqrt{\frac{k}{N}}
$$

and inequalities (7.4) follow.

We first prove an auxiliary estimate.

Lemma 7.4. Let $k \in\{0,1, \ldots, N-1\}, X^{\prime} \in \mathcal{D}_{k}, v \in X^{\prime}$ and $r_{j} \notin X^{\prime}$ be given such that $|v|=1$. Put

$$
\left.\begin{array}{l}
\eta_{0}=0 \\
\eta_{k}=1-\frac{1}{2}\left(1+\frac{1}{k}\right) \delta_{k+1}^{2} \quad(k=1, \ldots, N-1)
\end{array}\right\}
$$

Then

$$
\left|\left\langle r_{j}, v\right\rangle\right| \leq \eta_{k}
$$

Proof. The case $k=0$ is trivial. For $k \geq 1$ we find $J^{\prime} \in \mathcal{L}_{k}$ and real numbers $\alpha_{i} \quad\left(i \in J^{\prime}\right)$ such that $\operatorname{span}\left\{r_{i}: i \in J^{\prime}\right\}=X^{\prime}$ and $v=\sum_{i \in J^{\prime}} \alpha_{i} r_{i}$. We have indeed $J^{\prime \prime}:=J^{\prime} \cup\{j\} \in \mathcal{L}_{k+1}$ (note that Hypothesis 7.1 has been used here), and

$$
\left.\begin{array}{l}
1+|v|^{2}-2\left\langle r_{j}, v\right\rangle=\left|r_{j}-v\right|^{2} \geq \delta_{k+1}^{2}\left(1+\sum_{i \in J^{\prime}} \alpha_{i}^{2}\right) \geq\left(1+\frac{1}{k}\right) \delta_{k+1}^{2} \\
1+|v|^{2}+2\left\langle r_{j}, v\right\rangle=\left|r_{j}+v\right|^{2} \geq \delta_{k+1}^{2}\left(1+\sum_{i \in J^{\prime}} \alpha_{i}^{2}\right) \geq\left(1+\frac{1}{k}\right) \delta_{k+1}^{2}
\end{array}\right\}
$$

and the assertion follows

Let $\eta_{0}, \ldots, \eta_{N-1}$ be defined as in Lemma 7.4. For arbitrary $s \geq 0$ and $k=0, \ldots, N$ we define the sequence $M_{k}(s)$ by the recurrent formula

$$
\left.\begin{array}{l}
M_{0}(s)=0 \\
M_{k}^{2}(s)=M_{k-1}^{2}(s)+\frac{1}{1-\eta_{k-1}^{2}}\left(1+s+\eta_{k-1} M_{k-1}(s)\right)^{2}
\end{array}\right\} .
$$

Note that for all $s>0$ and $k=1, \ldots, N$

$$
\left(\frac{M_{k}(s)}{s}\right)^{2}=\left(\frac{M_{k-1}(s)}{s}\right)^{2}+\frac{1}{1-\eta_{k-1}^{2}}\left(\frac{1}{s}+1+\eta_{k-1} \frac{M_{k-1}(s)}{s}\right)^{2}
$$

hence each of the functions $s \mapsto \frac{M_{k}(s)}{s}(k=1, \ldots, N)$ is decreasing in $(0, \infty)$ and

$$
\lim _{s \rightarrow \infty} \frac{M_{k}(s)}{s}=M_{k}(0) \quad(k=1, \ldots, N) .
$$


For every $s \geq 0$ define a functional $V_{s}: X \rightarrow \mathbb{R}^{+}$by the formula

$$
V_{s}(z)=\max \left\{M_{k}^{2}(s)+\left|\left(P_{R_{J}}-P_{X^{\prime}}\right) z\right|^{2}: X^{\prime} \in \mathcal{D}_{k} \quad(k=0, \ldots, N-1)\right\}
$$

Obviously, $V_{s}$ is convex and the set

$$
B_{s}=\left\{z \in R_{J}: V_{s}(z) \leq M_{N}^{2}(s)\right\}
$$

is convex and closed for every $s \geq 0$.

Our main goal is to prove the following

Theorem 7.5. Let Hypothesis 7.1 hold. Assume moreover that

$$
\sigma:=\max _{j \in J}\left|n_{j}-r_{j}\right|<\frac{1}{M_{N}(0)}
$$

and let $s \geq 0$ satisfy the equation

$$
\frac{s}{M_{N}(s)}=\sigma
$$

Then the set $B=B_{\text {s }}$ defined by (7.12) satisfies condition $(\mathcal{B})$.

Indeed, from (7.10) it follows that condition (7.14) is meaningful and the value of $s$ is uniquely determined. Moreover, for every $z \in X$ we have

$$
V_{s}(z) \geq M_{0}^{2}(s)+\left|\left(P_{R_{J}}-P_{\{0\}}\right) z\right|^{2}=\left|P_{R_{J}} z\right|^{2}
$$

hence by $(7.12)$

$$
|z| \leq M_{N}(s) \quad(z \in B) .
$$

In particular, the set $B$ in Theorem 7.5 is contained in the ball centered at the origin with radius $M_{N}(s)$.

The proof of Theorem 7.5 is based on the following lemma.

Lemma 7.6. Let the hypotheses of Theorem 7.5 hold. Further, assume that for some $z \in B$ and $X^{\prime} \in \mathcal{D}_{k} \quad(k \in\{0, \ldots, N-1\})$ we have $M_{k}^{2}(s)+\left|\left(I-P_{X^{\prime}}\right) z\right|^{2}=M_{N}^{2}(s)$, and that there exists $i \in J$ such that $\left|\left\langle z, n_{i}\right\rangle\right|<1$. Then $r_{i} \in X^{\prime}$.

Proof. Assume $r_{i} \notin X^{\prime}$ and put $X^{\prime \prime}=X^{\prime} \oplus \operatorname{span}\left\{r_{i}\right\}$. We find $v \in X^{\prime}$ with $|v|=1$ and real numbers $a, b$ such that

$$
P_{X^{\prime \prime}} z=a r_{i}+b v
$$

Putting $\eta=\left\langle r_{i}, v\right\rangle \in\left[-\eta_{k}, \eta_{k}\right]$ we have

$$
\begin{aligned}
\left|P_{X^{\prime \prime}} z\right|^{2} & =a^{2}+b^{2}+2 a b \eta \\
\left|P_{X^{\prime}} z\right| & \geq\left|\left\langle P_{X^{\prime \prime}} z, v\right\rangle\right|=|a \eta+b|
\end{aligned}
$$


and, by hypothesis,

$$
|a+b \eta|=\left|\left\langle P_{X^{\prime \prime}} z, r_{i}\right\rangle\right|=\left|\left\langle z, r_{i}\right\rangle\right| \leq\left|\left\langle z, n_{i}\right\rangle\right|+|z|\left|n_{i}-r_{i}\right|<1+\sigma|z| .
$$

According to (7.14), we conclude from (7.20) and (7.16) that

$$
|a+b \eta|<1+s
$$

The assumption $z \in B$ moreover yields

$$
M_{k+1}^{2}(s)+\left|\left(I-P_{X^{\prime \prime}}\right) z\right|^{2} \leq M_{k}^{2}(s)+\left|\left(I-P_{X^{\prime}}\right) z\right|^{2}
$$

(note that for $k=N-1$ we have $\left(I-P_{X^{\prime \prime}}\right) z=0$ ), and we obtain

$$
M_{k+1}^{2}(s)-M_{k}^{2}(s) \leq\left|P_{X^{\prime \prime}} z\right|^{2}-\left|P_{X^{\prime}} z\right|^{2}
$$

where

$$
M_{k+1}^{2}(s)-M_{k}^{2}(s)=\frac{1}{1-\eta_{k}^{2}}\left(1+s+\eta_{k} M_{k}(s)\right)^{2}
$$

and

$$
\begin{aligned}
\left|P_{X^{\prime \prime}} z\right|^{2} & =(a \eta+b)^{2}+a^{2}\left(1-\eta^{2}\right) \\
& =(a \eta+b)^{2}+\frac{1}{1-\eta^{2}}(a+b \eta-\eta(a \eta+b))^{2} \\
& <\left|P_{X^{\prime}} z\right|^{2}+\frac{1}{1-\eta^{2}}\left(1+s+|\eta|\left|P_{X^{\prime}} z\right|\right)^{2} \\
& \leq\left|P_{X^{\prime}} z\right|^{2}+\frac{1}{1-\eta_{k}^{2}}\left(1+s+\eta_{k}\left|P_{X^{\prime}} z\right|\right)^{2}
\end{aligned}
$$

Combining (7.23) - (7.25) we obtain

$$
M_{k}(s)<\left|P_{X^{\prime}} z\right|
$$

hence

$$
M_{k}^{2}(s)+\left|\left(I-P_{X^{\prime}}\right) z\right|^{2}<|z|^{2} \leq M_{N}^{2}(s)
$$

which is a contradiction. Lemma 7.6 is proved

We now pass to the proof of Theorem 7.5.

Proof of Theorem 7.5. Assume $z \in B$ is given and $\left|\left\langle z, n_{i}\right\rangle\right|<1$ for some $i \in J$. For $\mu_{0}>0$ and $\mu \in\left[-\mu_{0}, \mu_{0}\right]$ put $z_{\mu}=z+\mu r_{i}$. Then $z_{\mu} \in R_{J}$ and for every $X^{\prime} \in$ $\mathcal{D}_{k}(k=1, \ldots, N-1)$ we either have $M_{k}^{2}(s)+\left|\left(I-P_{X^{\prime}}\right) z\right|^{2}=M_{N}^{2}(s)$, hence by Lemma $7.6 M_{k}^{2}(s)+\left|\left(I-P_{X^{\prime}}\right) z_{\mu}\right|^{2}=M_{N}^{2}(s)$, or $M_{k}^{2}(s)+\left|\left(I-P_{X^{\prime}}\right) z\right|^{2}<M_{N}^{2}(s)$, hence $\mu_{0}>0$ can be chosen in such a way that $z_{\mu} \in B$ for every $\mu \in\left[-\mu_{0}, \mu_{0}\right]$. For every $y \in \mathcal{N}_{B}(z)$ and every $\mu \in\left[-\mu_{0}, \mu_{0}\right]$ then $\left\langle y, z-z_{\mu}\right\rangle \geq 0$, hence $\left\langle y, r_{i}\right\rangle=0$ and Theorem 7.5 is proved 


\section{Example}

We illustrate our theory on an example motivated by a problem from queuing theory. Let us consider a service point, where two kinds of customers are served: the ordinary ones and the privileged ones. The waiting room has maximal capacity $K>0$, and the counters $O$ for ordinary and $P$ for privileged customers have maximal capacities $c_{o}$ and $c_{p}$, respectively.

The incoming customer flow can be described by a time-dependent vector $v(t)=$ $\left(\begin{array}{l}v_{o}(t) \\ v_{p}(t)\end{array}\right)$ where $v_{o}(t)$ and $v_{p}(t)$ represent the number of ordinary and privileged customers, respectively, arrived during the time interval $[0, t]$. We denote by $\eta(t)=\left(\begin{array}{l}\eta_{o}(t) \\ \eta_{p}(t)\end{array}\right)$ the respective number of customers who have left the service point during the interval $[0, t]$, and $x(t)=v(t)-\eta(t)=\left(\begin{array}{l}x_{o}(t) \\ x_{p}(t)\end{array}\right)$ is the queue at time $t$. The waiting room is then described by the condition

$$
x(t) \in Z:=\left\{\left(\begin{array}{l}
x_{o} \\
x_{p}
\end{array}\right): x_{o}, x_{p} \geq 0 \text { and } x_{o}+x_{p} \leq K\right\}
$$

for every $t \in[0, T]$ (see Figure 1$)$.

Figure 1: The Skorokhod diagram

We consider the following service rules:

A. All customers are served at their respective counters.

B. Both counters work at their maximal capacity.

C. If there is an unused capacity at the counter $O$, it can also be used by privileged customers.

D. If the capacity of the waiting room is exceeded, for each refused privileged customer there must be $\varrho$ refused ordinary customers for some $\varrho>0$.

This can be formalized in the following way: 
(i) $x_{o}>0, x_{p}>0, x_{o}+x_{p}<K \quad \Longrightarrow \quad \dot{\eta}_{o}=c_{o}, \dot{\eta}_{p}=c_{p}$.

(ii) $x_{o}>0, x_{p}=0, x_{o}+x_{p}<K \quad \Longrightarrow \quad \dot{\eta}_{o}=c_{o}, \dot{\eta}_{p}=\min \left\{c_{p}, \dot{v}_{p}\right\}$.

(iii) $x_{o}=0, x_{p}>0, x_{o}+x_{p}<K \quad \Longrightarrow \quad \dot{\eta}_{o}=\min \left\{c_{o}, \dot{v}_{o}\right\}, \dot{\eta}_{p}=c_{p}+c_{o}-\dot{\eta}_{o}$.

(iv) $x_{o}>0, x_{p}>0, x_{o}+x_{p}=K \quad \Longrightarrow \quad \dot{\eta}_{o}-c_{o}=\varrho\left(\dot{\eta}_{p}-c_{p}\right)$.

We normalize the problem by putting

$$
u(t)=v(t)-t\left(\begin{array}{c}
c_{o} \\
c_{p}
\end{array}\right), \quad \xi(t)=\eta(t)-t\left(\begin{array}{c}
c_{o} \\
c_{p}
\end{array}\right) .
$$

Hence we are in the situation of (1.7) - (1.8) with normal and reflection vectors

$$
\begin{aligned}
& n_{1}=\left(\begin{array}{c}
-1 \\
0
\end{array}\right), r_{1}=\frac{1}{\sqrt{2}}\left(\begin{array}{c}
-1 \\
1
\end{array}\right), n_{2}=r_{2}=\left(\begin{array}{c}
0 \\
-1
\end{array}\right) \\
& n_{3}=\frac{1}{\sqrt{2}}\left(\begin{array}{l}
1 \\
1
\end{array}\right), r_{3}=\frac{1}{\sqrt{\varrho^{2}+1}}\left(\begin{array}{l}
\varrho \\
1
\end{array}\right)
\end{aligned}
$$

(see Figure 1). The projections $Q_{j}$ have the form

$$
Q_{1}\left(\begin{array}{c}
x_{o} \\
x_{p}
\end{array}\right)=\left(\begin{array}{c}
x_{o} \\
-x_{o}
\end{array}\right), \quad Q_{2}\left(\begin{array}{c}
x_{o} \\
x_{p}
\end{array}\right)=\left(\begin{array}{c}
0 \\
x_{p}
\end{array}\right), \quad Q_{3}\left(\begin{array}{c}
x_{o} \\
x_{p}
\end{array}\right)=\frac{1}{\varrho+1}\left(x_{o}+x_{p}\right)\left(\begin{array}{c}
\varrho \\
1
\end{array}\right) .
$$

Using the identity

$$
\left(I-Q_{3}\right)\left(\begin{array}{l}
x_{o} \\
x_{p}
\end{array}\right)=\left(\begin{array}{c}
(1-\varepsilon) x_{o}-\varepsilon x_{p}-\left(\frac{\varrho}{\varrho+1}-\varepsilon\right)\left(x_{o}+x_{p}\right) \\
(1-\varepsilon(1+\varepsilon)) x_{p}-(1+\varepsilon) \varepsilon x_{o}-\left(\frac{1}{\varrho+1}-\varepsilon(1+\varepsilon)\right)\left(x_{o}+x_{p}\right)
\end{array}\right)
$$

and the triangle inequality we easily check that the system $\mathcal{Q}$ is $\ell$-paracontracting with respect to the norm

$$
\left\|\left(\begin{array}{c}
x_{o} \\
x_{p}
\end{array}\right)\right\|=C\left((1+\varepsilon)\left|x_{o}\right|+\left|x_{p}\right|+(1-\varepsilon)\left|x_{o}+x_{p}\right|\right)
$$

whenever $\varepsilon<\frac{\varrho}{\varrho+1}, \varepsilon(1+\varepsilon)<\frac{1}{\varrho+1}$ and $2 C \varepsilon^{2} \geq 1$. The transversality is indeed obvious. The construction of the set $B^{\omega}$ from Corollary 4.5 is shown on Figure 2.

Figure 2: Construction of the set $B^{\omega}$ 
In the limit case $\varrho=+\infty$ the system is not $\ell$-paracontracting any more, as

$$
\left(I-Q_{1}\right)\left(I-Q_{2}\right)\left(I-Q_{3}\right)\left(\begin{array}{c}
0 \\
x_{p}
\end{array}\right)=\left(\begin{array}{c}
0 \\
-x_{p}
\end{array}\right)
$$

for every $x_{p} \in \mathbb{R}$ which would contradict inequality (5.3). Similarly, for $\varrho=0$ we have $r_{3}=-r_{2}$, hence $R_{\{2,3\}}^{\perp} \cap N_{\{2,3\}} \neq\{0\}$ in contradiction with Lemma 5.2. In other words, difficulties arise if only ordinary customers or only privileged customers are refused in the case of exceeded capacity.

\section{References}

[1] Desch, W. and J. Turi: The stop operator related to a convex polyhedron. J. Diff. Equ. 157 (1999), $329-347$.

[2] Drábek, P., Krejčí, P. and P. Takáč: Nonlinear Differential Equations (Research Notes in Mathematics: Vol. 404). London: Chapman \& Hall/CRC 1999.

[3] Dupuis, P. and H. Ishii: On Lipschitz continuity of the solution mapping to the Skorokhod problem. Stoch. and Stoch. Reports 35 (1991), $31-62$.

[4] Dupuis, P. and K. Ramanan: Convex duality and the Skorokhod problem, Parts I and II. Probab. Theory Relat. Fields 115 (1999), 153 - 195 and $197-236$.

[5] Elsner, L. and S. Friedland: Norm conditions for convergence of infinite products. Lin. Alg. Appl. 250 (1997), $133-142$.

[6] Elsner, L., Koltracht, I. and M. Neumann: On the convergence of asynchronous paracontractions with applications to tomographic reconstructions from incomplete data. Lin. Alg. Appl. 130 (1990), $65-82$.

[7] Krasnosel'skii, M. A. and A. V. Pokrovskii: Systems with Hysteresis. Berlin - Heidelberg: Springer-Verlag 1988.

[8] Krejčí, P.: Hysteresis, Convexity and Dissipation in Hyperbolic Equations (Gakuto Int. Ser. Math. Sci. Appl.: Vol. 8). Tokyo: Gakkōtosho 1996.

[9] Vladimirov, A. A., Elsner, L. and W.-J. Beyn: Stability and paracontractivity of discrete linear inclusions. Lin. Alg. Appl. 312 (2000), 125 - 134.

[10] Vladimirov, A. A. and A. F. Kleptsyn: On some hysteresis elements (in Russian). Avtomat. i Telemekh. 1982(7), $165-169$.

Received 23.03.2000 